Publications of the Astronomical Society of the Pacific, 118: 1690-1710, 2006 December

(C) 2006. The Astronomical Society of the Pacific. All rights reserved. Printed in U.S.A.

\title{
The Formation and Evolution of Planetary Systems: Placing Our Solar System in Context with Spitzer
}

\author{
Michael R. Meyer, ${ }^{1}$ Lynne A. Hillenbrand, ${ }^{2}$ Dana Backman, ${ }^{3}$ Steve Beckwith,,${ }^{4,5}$ Jeroen Bouwman, ${ }^{6}$ Tim Brooke, ${ }^{2}$ \\ John Carpenter, ${ }^{2}$ Martin Cohen, ${ }^{7}$ Stephanie Cortes, ${ }^{1}$ Nathan Crockett, ${ }^{8}$ Uma Gorti, ${ }^{9}$ Thomas Henning, ${ }^{6}$ \\ Dean Hines, ${ }^{10}$ David Hollenbach, ${ }^{9}$ Jinyoung Serena Kim, ${ }^{1}$ Jonathan Lunine, ${ }^{10}$ Renu Malhotra, ${ }^{11}$ Eric Mamajek, ${ }^{12}$ \\ Stanimir Metchev, ${ }^{13}$ Amaya Moro-Martin, ${ }^{14}$ Pat Morris,${ }^{15}$ Joan Najita, ${ }^{8}$ Deborah Padgett,${ }^{16}$ Ilaria Pascucci,,${ }^{1}$ \\ Jens Rodmann, ${ }^{6}$ Wayne Schlingman, ${ }^{1}$ Murray Silverstone, ${ }^{1}$ David Soderblom, ${ }^{4}$ John Stauffer, ${ }^{16}$ Elizabeth Stobie, ${ }^{1}$ \\ Steve Strom, ${ }^{8}$ Dan Watson, ${ }^{17}$ Stuart Weidenschilling, ${ }^{18}$ Sebastian Wolf, ${ }^{6}$ and Erick Young ${ }^{1}$ \\ Received 2006 May 30; accepted 2006 October 10; published 2006 December 18
}

\begin{abstract}
We provide an overview of the Spitzer Legacy Program, Formation and Evolution of Planetary Systems, that was proposed in 2000, begun in 2001, and executed aboard the Spitzer Space Telescope between 2003 and 2006. This program exploits the sensitivity of Spitzer to carry out mid-infrared spectrophotometric observations of solar-type stars. With a sample of $\sim 328$ stars ranging in age from $\sim 3$ Myr to $~ 3$ Gyr, we trace the evolution of circumstellar gas and dust from primordial planet-building stages in young circumstellar disks through to older collisionally generated debris disks. When completed, our program will help define the timescales over which terrestrial and gas giant planets are built, constrain the frequency of planetesimal collisions as a function of time, and establish the diversity of mature planetary architectures. In addition to the observational program, we have coordinated a concomitant theoretical effort aimed at understanding the dynamics of circumstellar dust with and without the effects of embedded planets, dust spectral energy distributions, and atomic and molecular gas line emission. Together with the observations, these efforts will provide an astronomical context for understanding whether our solar system - and its habitable planet-is a common or a rare circumstance. Additional information about the FEPS project can be found on the team Web site.
\end{abstract}

\section{INTRODUCTION}

The Spitzer Space Telescope (Werner et al. 2004), formerly SIRTF (Space Infrared Telescope Facility), is an $85 \mathrm{~cm}$ cry-

\footnotetext{
${ }^{1}$ Steward Observatory, University of Arizona, 933 North Cherry Avenue, Tucson, AZ 85721-0065; mmeyer@as.arizona.edu.

${ }^{2}$ California Institute of Technology, Pasadena, CA.

${ }^{3}$ Stratospheric Observatory for Infrared Astronomy, and SETI Institute, Mountain View, CA.

${ }^{4}$ Space Telescope Science Institute, 3700 San Martin Drive, Baltimore, MD 21218.

5 Department of Physics and Astronomy, Johns Hopkins University, Baltimore, MD.

${ }^{6}$ Max-Planck-Institut für Astronomie, Heidelberg, Germany.

${ }^{7}$ Radio Astronomy Laboratory, University of California, Berkeley, CA.

${ }^{8}$ National Optical Astronomy Observatories, Tucson, AZ.

${ }^{9}$ NASA-Ames Research Center, Theory Branch, Moffett Field, CA.

${ }^{10}$ Space Science Institute, 4750 Walnut Street, Suite 205, Boulder, CO 80301.

${ }^{11}$ Lunar and Planetary Laboratory, University of Arizona, Tucson, AZ

${ }^{12}$ Harvard-Smithsonian Center for Astrophysics, Cambridge, MA.

${ }^{13}$ University of California, Los Angeles, CA.

${ }^{14}$ Department of Astrophysical Science, Princeton University, Princeton, NJ.

${ }^{15}$ Herschel Science Center, IPAC, California Institute of Technology, Pasadena, CA.

${ }^{16}$ Spitzer Science Center, California Institute of Technology, Pasadena, CA.

${ }^{17}$ Department of Physics and Astronomy, University of Rochester, Rochester, NY.

${ }^{18}$ Planetary Science Institute, Tucson, AZ.
}

ogenic space observatory in Earth-trailing orbit. The observatory was launched in 2003 August and has an estimated mission lifetime of $5+$ years. There are three science instruments on board: the Infrared Array Camera (IRAC; Fazio et al. 2004), the Infrared Spectrograph (IRS; Houck et al. 2004), and the Multiband Imaging Photometer for Spitzer (MIPS; Rieke et al. 2004), which together provide the capability for imaging and spectroscopy from 3.6 to $160 \mu \mathrm{m}$. The Legacy Science Program was established before launch with two goals: to enable large-scale programs of broad scientific and public interest, and to provide access to uniform and coherent data sets as rapidly as possible, in support of General Observer (GO) proposals, given the limited lifetime of the mission. The Formation and Evolution of Planetary Systems (FEPS) ${ }^{19}$ Spitzer Legacy Science Program is one of six such original programs (for descriptions of the others, see Evans et al. 2003; Benjamin et al. 2003; Kennicutt et al. 2003; Lonsdale et al. 2003; Dickinson et al. 2003) and uses 350 hours of Spitzer observing time. FEPS builds on the rich heritage of Spitzer's ancestors in space- the international all-sky mid-infrared survey telescope IRAS (Infrared Astronomical Satellite, 1983-1985) and the European Space Agency's (ESA's) pointed mission ISO (Infrared

\footnotetext{
${ }^{19}$ See http://feps.as.arizona.edu.
} 
Space Observatory, 1995-1999)—and complements Guaranteed Time Observer (GTO) and GO programs also being pursued with Spitzer.

In a single sentence, FEPS is a comprehensive study of the evolution of gas and dust in the circumstellar environment. The scientific motivation for FEPS lies in the fragmented but compelling evidence for dusty circumstellar material surrounding stars spanning a wide range of ages, from young pre-mainsequence stars to those as old as, and even older than, the Sun.

At young ages, incontrovertible evidence assembled over the past three decades (based on data from ultraviolet through millimeter wavelengths) suggests that most stars are surrounded at birth by accretion disks that are remnants of the star formation process itself (e.g., Beckwith \& Sargent 1996). The revelations provided by $I R A S$, and later $I S O$, led to a nearly complete census of optically thick disks within 100-200 pc, and in the case of $I S O$ revealed their rich dust mineralogy and gas content (see Lorenzetti [2005] and Molster \& Kemper [2005] for reviews). That at least some of these disks build planets has become clear from radial velocity and photometric studies revealing $M \sin i=0.02 M_{\text {Jup }}-15 M_{\text {Jup }}$ planets orbiting well over 100 nearby stars (e.g., Marcy et al. 2005). At older ages, IRAS and ISO revealed the presence around dozens of main-sequence stars of micron-sized grains. These dusty "debris" disks are produced in collisions between asteroid-like bodies with orbits that are dynamically stirred by planets (e.g., Lagrange et al. 2000). Subsequently, several of these disks were spatially resolved at optical, infrared, and millimeter wavelengths (e.g., Kalas et al. 2004; Weinberger et al. 1999; Greaves et al. 1998), revealing structure consistent with the planetary perturber interpretation.

The connections among planets, debris disks, and the dusty and gaseous disks nearly ubiquitously found in association with recently formed young stars are tantalizing, but not yet unequivocally established. Understanding the evolution of young circumstellar dust and gas disks as they transition through the planet-building phase requires the 100-fold enhancement in sensitivity and increased photometric accuracy offered by Spitzer at mid- and far-infrared wavelengths. For main-sequence stars, while IRAS discovered the prototypical debris disks (see Backman \& Paresce [1993] for a review) and ISO made additional surveys (see de Muizon [2005] for a review), neither IRAS nor ISO was sensitive enough to detect dust in solar systems older than a few hundred Myr for any but the nearest tens of stars. ${ }^{20}$ Spitzer, by contrast, can detect ordersof-magnitude-smaller dust masses: for a solar-type star at $30 \mathrm{pc}$, down to $\sim 10^{20} \mathrm{~kg}$ or $\sim 10^{-5} M_{\oplus}$ in micron- to submillimeter-size grains at $50 \mathrm{~K}$, only an order of magnitude above the dust mass inferred for our own present-day Kuiper Belt, and $\sim 10^{17} \mathrm{~kg}$ or $\sim 10^{-8} M_{\oplus}$ at $150 \mathrm{~K}$, only an order of magnitude above the dust mass in our present-day asteroid belt plus zodiacal cloud.

\footnotetext{
${ }^{20}$ The IRAS and ISO observatories were able to study representative samples of A-type stars, but not G-type stars.
}

The FEPS program is designed to study circumstellar dust properties around a representative sample of solar-type stars. Included are 328 stars chosen to probe the suspected direct link between disks commonly found around pre-main-sequence stars less than 3.0 Myr old and our 4.56 Gyr old Sun and solar system. Specifically, we trace the evolution of circumstellar material at ages 3-10 Myr when stellar accretion from the disk terminates, to 10-100 Myr when planets achieve their final masses via coalescence of solids and accretion of remnant molecular gas, to $100-1000 \mathrm{Myr}$ when the final architecture of solar systems takes form and frequent collisions between remnant planetesimals produce copious quantities of dust, and finally, to 1-3 Gyr mature systems in which the planet-driven activity of planetesimals continues to generate detectable dust. Our sample is distributed uniformly in log-age from 3 Myr to $3 \mathrm{Gyr}$. We probe the full range of the dust disk optical-depth diagnostic of the major phases of planet system formation and evolution, including primordial disks (those dominated by interstellar medium [ISM] grains in the process of agglomerating into planetesimals) and debris disks (those dominated by collisionally generated dust) like our own.

Our strategy is to obtain for all 328 stars in our sample carefully calibrated spectral energy distributions (SEDs), using all three Spitzer instruments. A high-resolution spectroscopic survey limited to the younger targets establishes the gas content. In addition to insight into problems of fundamental scientific and philosophical interest, the FEPS Legacy Science Program provides a rich database for follow-up observations with Spitzer, with existing and future ground-based facilities, as well as with the SIM PlanetQuest (Space Interferometry Mission PlanetQuest), the JWST (James Webb Space Telescope), and eventually the TPF (Terrestrial Planet Finder). FEPS complements and motivates many existing Spitzer GTO and GO programs.

\section{SCIENCE STRATEGY}

We take advantage of Spitzer's unprecedented mid-infrared sensitivity and hence its unique ability to detect the photospheres of solar-type stars out to distances of several tens of pc. Spitzer observations of excesses above those photospheres are indicative of dust located at a range of orbital separations, from analogs of the terrestrial zone, to the gas-giant zone, to the Kuiper Belt zone in our own solar system. Such observations are important in the search for exosolar planetary systems-either those in formation from primordial dust and gas disks, or those that later perturb planetesimals into crossing orbits that collisionally cascade to produce dusty debris disks. For a given system, the mass in small grains to which Spitzer is sensitive is first expected to decrease with time as planet formation begins, then increase on a relatively rapid timescale (few Myr) as the debris phase begins, and finally decrease on a much longer timescale (many Gyr) as the disk slowly grinds itself down and grains are removed via radiative and mechanical effects. 
With the goal of understanding how common or rare the evolutionary path taken by our solar system might have been, we have initiated a Spitzer survey of F-G-K (solar-type) stars. First, we study the formation of planetary embryos in a survey of postaccretion circumstellar dust disks. We aim to understand the evolution of disk properties (mass and radial structure) and dust properties (size and composition) during the main phase of planet-building and early solar system evolution from 3 to 100 Myr. Second, we study the growth of gas giants in a sensitive search for warm molecular gas at the level of $>2 \times$ $10^{-4} M_{\odot}\left[\mathrm{H}_{2}\right]$ at $70-200 \mathrm{~K}$, in a subsample of the targets from our dust disk survey. Our goal is to constrain directly the time available for embryonic planets to accrete gas envelopes. Third, we investigate mature solar system evolution by tracing 100 Myr to 3 Gyr old dust disks generated through collisions of planetesimals. Through our analysis, we hope to infer the locations and masses of giant planets greater than $0.05 M_{\text {Jup }} \approx$ $1 M_{\text {Uranus }}$ through their action on the remnant disk.

Our large sample enables us to measure the mean properties of evolving dust disks and discover the dispersion in evolutionary timescales. Further, we can search for relations between inferred dust evolutionary path and stellar properties such as metallicity and multiplicity. In the following subsections, we detail our science strategy.

\subsection{Formation of Planetary Embryos}

Our experiment begins as the disks are making the transition from optically thick to thin, the point at which all of the disk's mass first becomes detectable through observation. The FEPS goals are to:

1. Constrain the initial structure and composition of postaccretion, optically thin disks.

2. Trace the evolution of disk structure, composition, and mass over time.

3. Characterize the timescales over which primordial disks dissipate and debris disks arise.

4. Measure changes in the dust particle size distribution due to coagulation of interstellar grains at early stages and shattering associated with high-speed planetesimal collisions at later early-debris stages.

5 . Infer the presence of newly formed planets at orbital radii of $0.3-30$ AU.

Photometric observations from 3.6 to $160 \mu \mathrm{m}$ with Spitzer probe temperatures (radii) encompassing the entire system of planets in our solar system. In Figure 1, we show the mass sensitivity of Spitzer as a function of wavelength, indicating the mass in small grains that Spitzer can detect as a function of orbital radius. Detailed spectrophotometry in the range 5.3$40 \mu \mathrm{m}$ permits a search for gaps in disks caused by the dynamical interaction of young gas giant planets and the particulate disk from 0.2 to $10 \mathrm{AU}$. This extends from just outside the innermost radii of the exosolar "hot Jupiters" (thought to have suffered significant orbital migration in a viscous accretion disk) to the gas giants of our solar system (thought to have formed beyond the "ice line").

Mid-infrared spectroscopic observations are sensitive to dust properties, including size distribution and composition. They thus probe physical conditions in the disk. From observations in the 5.3-40 $\mu \mathrm{m}$ spectral region, we determine the relative importance of broad features attributed to amorphous silicates (ubiquitous in the ISM) compared to numerous narrow features due to crystalline dust (observed only in circumstellar environments). In this way, we can look for evidence of, e.g., radial mixing in the disk, since the temperature required to anneal grains $(>1000 \mathrm{~K})$ is substantially higher than that inferred for the continuum-emitting material $(\sim 300 \mathrm{~K})$. Further, the shape and strength of specific mid-infrared spectroscopic features provide constraints on the fractional contribution of each grain population to the total opacity, necessary for estimating dust mass surface densities (see $\S 4.2$ ).

\subsection{Growth of Gas Giants}

Next, we have undertaken the most sensitive survey to date of atomic and molecular gas in postaccretion disk systems. In order to characterize gas dissipation and to place limits on the time available for giant planet formation, we obtain high spectral resolution $(\mathcal{R}=600)$ data from 10 to $37 \mu \mathrm{m}$ with the $I R S$ of 35 stars selected from our dust disk survey sample. The data include the $\mathrm{S}(0) 28.2 \mu \mathrm{m}, \mathrm{S}(1) 17.0 \mu \mathrm{m}$, and $\mathrm{S}(2) 12.3 \mu \mathrm{m} \mathrm{H}_{2}$ lines, as well as strong atomic lines such as [S I] $25.23 \mu \mathrm{m}$, [Si II] $34.8 \mu \mathrm{m}$, and [Fe II] $26 \mu \mathrm{m}$ (Gorti \& Hollenbach 2004; Hollenbach et al. 2005). We focus on the postaccretion epochs from 3 to $100 \mathrm{Myr}$ to examine whether gas disks persist after disk accretion onto the star has ceased and planetesimal agglomeration has removed the dust disk, potentially providing "nucleation sites" for gas giant planet formation.

Understanding gas-dust dynamics is crucial to our ability to derive the timescales important in planet formation and evolution. The dust and gas experiments being conducted at young ages ( $<100 \mathrm{Myr})$ have an important synergy in furthering this understanding, because dust dynamics are controlled by gas drag rather than radiation pressure when the gas-to-dust mass ratio is greater than 0.1 , while it is the presence of dust that mediates gas heating and therefore detectability. If the gas-todust ratio is low (i.e., the dust opacity per gas particle is high) and the gas and dust are at similar temperatures, the detection of gas lines by Spitzer becomes difficult, due to the small ratio of line to continuum. However, the theoretical models of Gorti \& Hollenbach (2004) show that in many instances the dust opacity is sufficiently low and the gas temperature sufficiently high $(>100 \mathrm{~K})$ that small quantities $\left(<0.1 M_{\text {Jup }}\right)$ of gas, if present, can be detected by Spitzer around nearby disks with optically thin dust (see $\S 4.4$ ).

\subsection{Mature Solar System Evolution}

Finally, we conduct a study of second-generation "debris disks." The presence of any observable circumstellar dust 


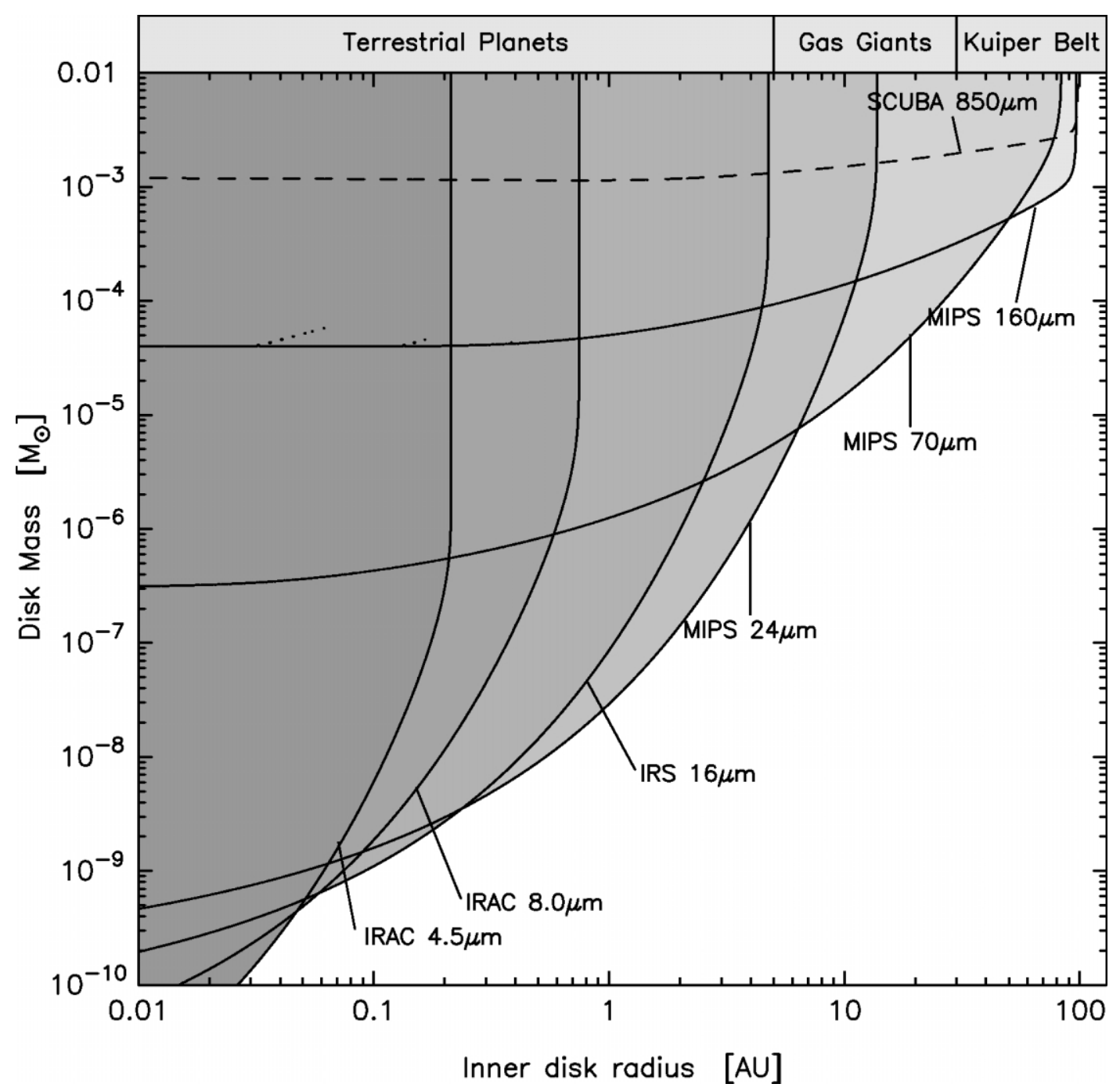

FIG. 1.-Spitzer sensitivity to mass in small grains, as a function of radius in a hypothetical circumstellar disk surrounding a Sun-like star at a distance of $30 \mathrm{pc}$ for integration times typical for FEPS. For emission from small grains in radiative equilibrium, each radius in the disk corresponds to a specific dust temperature. From simple blackbody considerations, shorter Spitzer wavelengths probe warmer dust (at smaller orbital radii), while longer Spitzer wavelengths probe cooler dust (at larger orbital radii), as indicated.

around stars older than the maximum lifetime of a primordial dust disk (the sum of the to-be-determined gas dissipation timescale and the characteristic Poynting-Robertson drag timescale) provides compelling evidence not only for large reservoirs of planetesimals colliding to produce the dust, but also for the existence of planetary bodies that dynamically perturb planetesimal orbits inducing frequent collisions.

We have undertaken the first comprehensive survey of F5K5 stars with ages $100 \mathrm{Myr}$ to $3 \mathrm{Gyr}$ that is sensitive to dust disks comparable to those characteristic of our own solar system throughout its evolution. We chart the history of our solar system from 100 to $300 \mathrm{Myr}$, the last phase of terrestrial and ice giant (Uranus- and Neptune-like) planet-building; through $0.3-1$ Gyr, bracketing the "late heavy bombardment" impact peak that might have had an effect on the early evolution of life on Earth; and finally over 1.0-3.0 Gyr, examining the diversity of evolutionary paths among a mature planetary system. Spectroscopic observations in the range 5.3-40 $\mu \mathrm{m}$ enable diagnosis of gaps caused by giant planets, and estimates of dust size and composition that translate directly into constraints on the mass opacity coefficients for the dust (Miyake \& Nakagawa 1993) as well as Poynting-Robertson drag timescales (Backman \& Parsece 1993).

\section{SURVEY PREPARATION AND EXECUTION}

\subsection{Observing Strategy}

A complete and uniform set of Spitzer photometric and spectroscopic observations are obtained for all stars in our dust disk evolution sample, as described below. To derive 
statistically meaningful results on the disk and dust properties, we observe $\sim 50$ stars in each of six logarithmically spaced age bins from $3 \mathrm{Myr}$ (connecting our legacy program to that of Evans et al. 2003) to 3 Gyr (beyond which there is strong emphasis by GTOs on debris disk science; Beichman et al. 2005). Our targets span a narrow mass range $\left(0.7-2.2 M_{\odot}\right)$ and are proximate enough to enable a complete census for circumstellar dust, comparable to our model solar system as a function of age. We measure the stellar photosphere at $\mathrm{S} / \mathrm{N}$ $>30$ for 3.6-24 $\mu \mathrm{m}$, and $\mathrm{S} / \mathrm{N}>5$ at $70 \mu \mathrm{m}$ (or $\mathrm{S} / \mathrm{N}>5$ for 5 times the current zodiacal dust emission) with broadband photometry from IRAC and MIPS (subject to calibration uncertainties). To identify gaps in the dust distribution created by the presence of giant planets from 0.2 to $10 \mathrm{AU}$, we require relative spectrophotometry with $\mathrm{S} / \mathrm{N}>30$ from 5.3 to $11 \mu \mathrm{m}$, and with $\mathrm{S} / \mathrm{N} \sim 6-12$ at wavelengths between 20 and $30 \mu \mathrm{m}$ with the IRS.

A subsample of 35 stars comprises our gas disk evolution study with the high-resolution mode of the IRS. This sample spans a range of spectral type (F3-K5), age (3-100 Myr), activity $\left(L_{x} / L_{\text {bol }} \sim 10^{-3}\right.$ to $\left.-10^{-5}\right)$, and a wide range of infrared excess emission, with some preference for optically thin excess in the mid-infrared. Fourteen stars were chosen for first-look observations and enable us to explore the limits implied by null results and guide our choice of follow-up observations for additional stars drawn from our dust disk survey.

Our goal is to collect data capable of realizing the fundamental limits imposed by instrument stability and systematic calibration uncertainties. Integration times are chosen according to each star's distance, age, and spectral type to reach a uniform $\mathrm{S} / \mathrm{N}$ at the photospheric limits, as specified above-thereby providing a complete census of dust disks for our targets.

\subsection{Sample Selection}

The source list for FEPS consists of young, near-solar analogs, stars ranging in mass from 0.7 to $2.2 M_{\odot}$ although strongly peaked at $1 M_{\odot}$ (see Fig. 2), and stars spanning ages 3 Myr to 3 Gyr (our Sun is 4.56 Gyr old). The stars are drawn from three recently assembled samples.

First, Soderblom \& King (1999) have produced a well-characterized set of $\sim 5000$ solar-type stars spread over the entire sky (see also Henry et al. 1996), having parallaxes that place the stars within $60 \mathrm{pc}, B-V$ colors between 0.52 and 0.81 (F8-K0 spectral types), and location in the Hertzsprung-Russell diagram within $1.0 \mathrm{mag}$ of the solar-metallicity zero-age main sequence. This sample is fully complete out to $50 \mathrm{pc}$. The age distribution in such a volume-limited region around the Sun is roughly flat in linear age units out to $2.5 \mathrm{Gyr}$, at which point heating by the Galactic disk has increased the scale height of older stars and thus removed them from the volumelimited sample. From this catalog, we have selected a sample with ages based on the $R^{\prime} H K$ chromospheric activity index from $\sim 100 \mathrm{Myr}$ to $3 \mathrm{Gyr}$. However, being located more than $100 \mathrm{pc}$ from the nearest sites of recent star formation, the

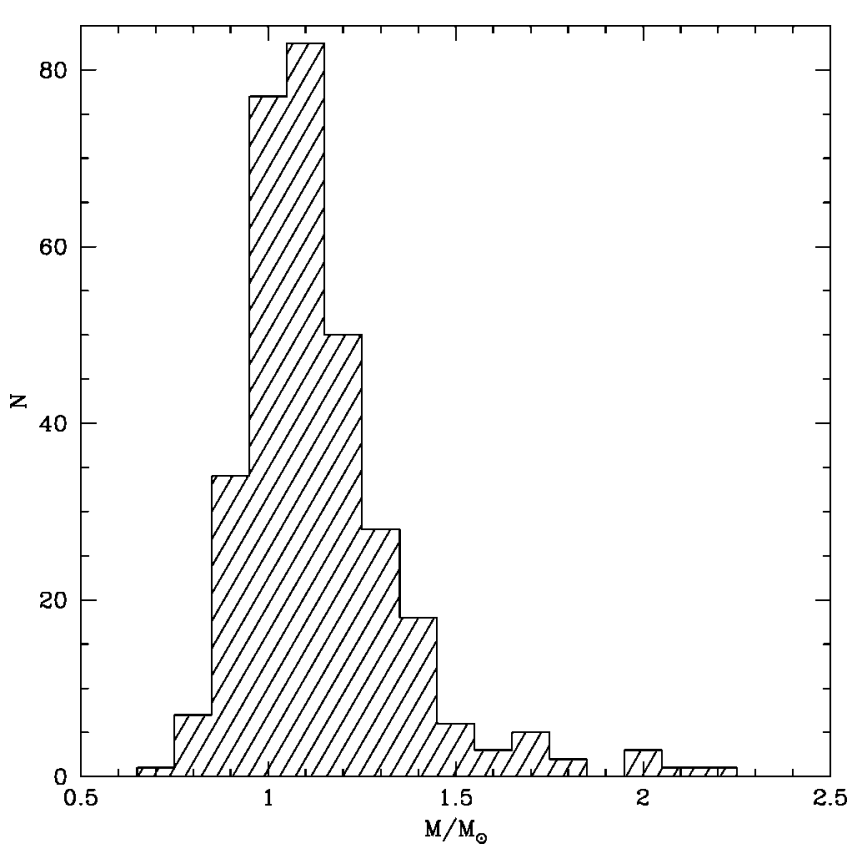

FIG. 2.-Distribution of masses for stars in the FEPS sample. The range spans $0.7-2.2 M_{\odot}$, although it is strongly peaked at $1.0 M_{\odot}$.

immediate solar neighborhood is deficient in stars with ages younger than 100 Myr. Hence, the volume limit was extended in order to identify large enough samples of young stars for the FEPS project.

We have conducted a new (e.g., Mamajek et al. 2002) and literature-based examination to identify stars whose ages are in the range 3-100 Myr. These were selected as having $B-$ $V$ colors between 0.58 and 1.15 or spectral types $\mathrm{G} 0-\mathrm{K} 0$, strong $\mathrm{X}$-ray emission, kinematics appropriate for the young Galactic disk, and high lithium abundance compared to the $120 \mathrm{Myr}$ old Pleiades. Young stars are copious coronal X-ray emitters, and a large body of literature demonstrates the connection among X-ray emission, chromospheric activity, stellar rotation, and age. The surface density distribution of X-ray sources detected by the Röntgensatellit (ROSAT) all-sky survey reveals a concentration of objects coincident with the Gould Belt, a feature in the distant solar neighborhood (50-1000 pc) comprised of an expanding ring of atomic and molecular gas of which nearly all star-forming regions within $1 \mathrm{kpc}$ are a part. These X-ray sources are thought to be the dispersed low-mass counterparts to a series of 1-100 Myr old open clusters and extant and fossil OB associations that delineate the Gould Belt (e.g., Torra et al. 2000; Guillout et al. 1998). Proper motion data enable us to select the nearest of these young, X-rayemitting stars with space motions consistent with those of higher mass stars having measured parallax, and hence estimate their distances. Follow-up optical spectroscopy of these X-ray + proper motion selected stars is used to confirm youth and determine photospheric properties. A total of $\sim 600$ field stars are X-ray-selected candidate young stars. 


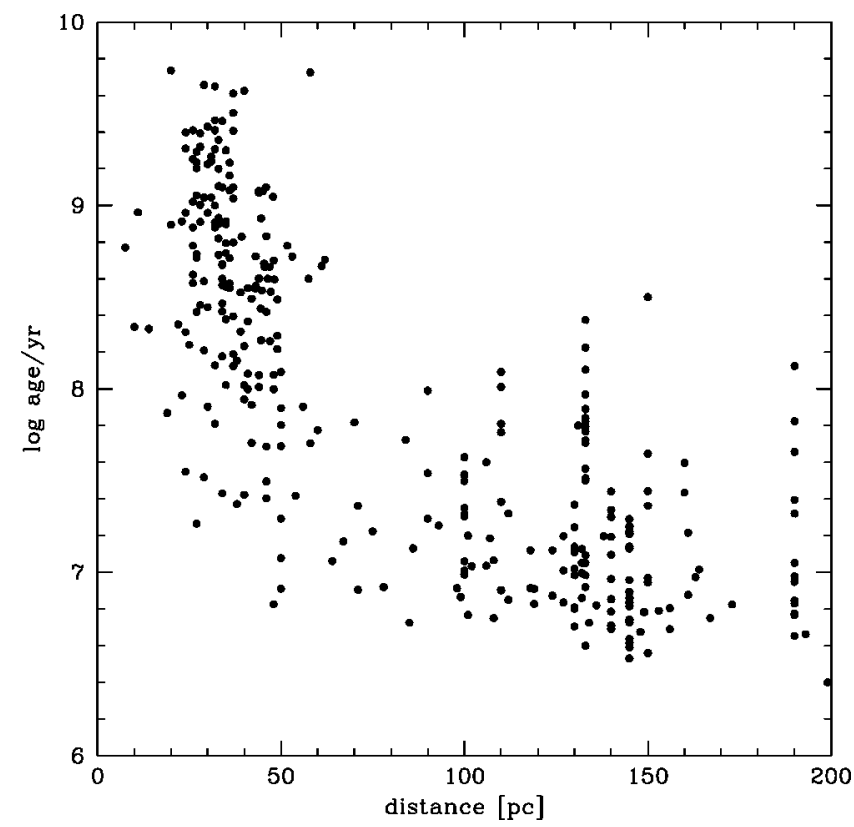

FIG. 3.-Estimated age vs. distance for all stars in the FEPS sample, comprised of nearby field stars, open cluster members, and young association members. Typical errors in age are less than $0.5 \mathrm{dex}$, while typical errors in distance are less than $10 \%$ for stars within $50 \mathrm{pc}$, and within $30 \%$ for more distant targets.

Finally, stars in nearby well-studied open clusters (IC 2602 [55 Myr], $\alpha$ Per [90 Myr], Pleiades [125 Myr], and Hyades [650 Myr]) serve to "benchmark" our field star results by providing samples nearly identical in age, composition, and birth environment. We considered all known members of these clusters meeting our targeted mass, $B-V$ color, and spectral type range that were not part of GTO samples.

From this large parent sample, stars were selected for potential observation with Spitzer if they met all of the following additional criteria. The criteria were chosen to ensure sufficient signal-to-noise ratio $(\mathrm{S} / \mathrm{N})$ on the stellar photosphere out to $24 \mu \mathrm{m}$ with Spitzer and thus accurate characterization of the underlying photosphere both observationally and with stellar models:

1. $K<10$ mag (young, $<100 \mathrm{Myr}, \mathrm{X}$-ray-selected and cluster samples) or $K<6.75$ mag (older, 0.1-3 Gyr, Hipparcos + $R^{\prime} H K$ selected sample).

2. $24 \mu \mathrm{m}$ background less than $1.70 \mathrm{mJy} \operatorname{arcsec}^{-2}$ (X-rayselected samples) or less than $1.54 \mathrm{mJy} \operatorname{arcsec}^{-2}$ (Hipparcos + $R^{\prime} H K$ sample).

3. $70 \mu \mathrm{m}$ background less than $0.76 \mathrm{mJy} \operatorname{arcsec}^{-2}$.

4. Galactic latitude $|b|>5^{\circ}$ (stars in IC 2602 were permitted to violate this criterion).

5. Quality Two Micron All Sky Survey (2MASS) JHK photometry, with no flags.

6. No projected 2MASS companions closer than $5^{\prime \prime}$.

7. No projected $2 \mathrm{MASS}$ companions closer than $15^{\prime \prime}$ unless they are both bluer in $J-K$ and fainter by $>3$ mag in $K$ than the Spitzer target.

These criteria were applied uniformly to our parent sample, although in the cases of a few exceptional stars (such as the IC 2602 sample), some criteria were violated. Next, targets appearing on Spitzer GTO programs were removed from the source list. Also, to a limited degree, stars identified through spectroscopy or high-resolution imaging literature published through 2001 March as being binary, with companions closer than $2^{\prime \prime}$, were removed. These cases were all either spectroscopic binaries or visual binaries with small delta magnitudes, and the literature search was not exhaustive. Subsequent investigation using adaptive optics (AO) imaging has uncovered additional binary systems with larger delta magnitudes remaining within the FEPS sample (e.g., Metchev \& Hillenbrand 2004).

Finally, among the stars in our parent sample older than $\sim 600 \mathrm{Myr}$, approximately $\frac{1}{2}$ were arbitrarily removed from our program in order to even out the age bins and bring the observing program within the allocated number of Spitzer hours (350).

Based on pre-Spitzer SEDs assembled from the literature, 2MASS, IRAS, ISO, and ancillary observations conducted to date, several (5-7) of the youngest stars in our program show some hint of circumstellar material. Because the stars were randomly selected based on their kinematic and activity properties as derived from optical information, these previously known dust excesses do not bias the program.

To this source list a set of 14 stars was added, which were suspected to have optically thin dust excesses based on observations from IRAS and ISO. These stars formed part of our first-look gas-disk detection survey and should not be used to derive the statistics of dust debris as a function of age.

Our final target list for observations with Spitzer and groundbased ancillary programs consists of 328 solar-type stars distributed uniformly in log-age between 3 Myr and 3 Gyr. Approximately 60 of these are members of the open clusters IC 2602, $\alpha$ Per, Pleiades, or Hyades. The remainder are field stars distributed in distance between 11 and $180 \mathrm{pc}$. The relation between distance and age for this sample is shown in Figure 3. The complete source list is presented in Table 1 (field stars), Table 2 (open cluster stars), Table 3 (young stars), and Table 4 (pre-selected IRS high-resolution targets). The names, coordinates, and spectral types are those found in the Spitzer Legacy Science Archive, ${ }^{21}$ and details will be presented in L. A. Hillenbrand et al. (2006, in preparation).

\subsection{Spitzer Data}

\subsubsection{Astronomical Observing Requests}

FEPS uses all three science instruments on board Spitzer to provide data from 3.6 to $70 \mu \mathrm{m}$ (with a subset of the FEPS

\footnotetext{
${ }^{21}$ See http://ssc.spitzer.caltech.edu/legacy/all.html.
} 
TABLE 1

Field STARS

\begin{tabular}{|c|c|c|c|}
\hline Name & R.A. (J2000.0) & Decl. (J2000.0) & Spectral Type \\
\hline HD 224873 & 000123.66 & +393638.12 & K0 \\
\hline HD $377^{\mathrm{a}}$ & $00 \quad 08 \quad 25.74$ & +06 3700.50 & $\mathrm{G} 2 \mathrm{~V}$ \\
\hline HD 691. & 001122.44 & +30 2658.52 & K0 V \\
\hline HD 984 . & $\begin{array}{llll}00 & 14 & 10.25\end{array}$ & -071156.92 & F7 V \\
\hline HD 6434 & 010440.15 & -392917.61 & $\mathrm{G} 2 / 3 \mathrm{~V}$ \\
\hline HD 6963 & $01 \quad 1041.91$ & +425554.50 & G7 V \\
\hline HD 7661 & $01 \quad 1624.19$ & -120549.33 & K0 V \\
\hline HIP 6276 & 012032.27 & -112803.74 & G0 \\
\hline HD 8941 & $\begin{array}{lll}01 & 2824.36\end{array}$ & +1704 45.20 & F8 IV-V \\
\hline HD 9472 & $\begin{array}{llll}01 & 33 & 19.03\end{array}$ & +23 5832.19 & G0 \\
\hline HD 11850 & 015647.27 & +230304.09 & G5 \\
\hline HD $12039^{\mathrm{a}} \ldots \ldots \ldots$ & 015748.98 & -215405.32 & $\mathrm{G} 3 / 5 \mathrm{~V}$ \\
\hline HD $13382 \ldots \ldots \ldots$ & $02 \quad 1123.15$ & +212238.39 & G5 V \\
\hline HD 13507 & $02 \quad 1255.00$ & +404006.00 & G5 V \\
\hline HD $13531 \ldots$ & $\begin{array}{lll}02 & 13 & 13.35\end{array}$ & +403027.34 & G7 V \\
\hline HD $13974 \ldots \ldots \ldots$ & $\begin{array}{llll}02 & 17 & 03.23\end{array}$ & +34 1327.32 & G0 V \\
\hline HD $18940 \ldots \ldots \ldots$ & 030328.65 & +230341.19 & G0 \\
\hline HD $19019 \ldots \ldots \ldots$ & 030350.82 & +06 0759.82 & F8 \\
\hline HD $19668^{\mathrm{a}} \ldots \ldots$ & 030942.28 & -093446.46 & G8/K0 V \\
\hline HD $21411 \ldots \ldots$. & 032611.11 & -303704.13 & G8 V \\
\hline HD $26990 \ldots \ldots \ldots$ & 041616.50 & +070934.15 & G0 (V) \\
\hline HD $27466 \ldots \ldots \ldots$ & 041957.08 & -042619.60 & G5 V \\
\hline HD 28495 & 043354.23 & +64 3759.40 & G0 \\
\hline HD $29231 \ldots \ldots \ldots$ & 043438.49 & -353929.06 & G8 V \\
\hline HD $31143 \ldots \ldots \ldots$ & 045145.71 & -355024.97 & K0 V \\
\hline HD $31392 \ldots \ldots$. & 045404.21 & -352416.28 & $\mathrm{~K} 0 \mathrm{~V}$ \\
\hline HD $32850 \ldots \ldots$ & 050642.21 & +142646.42 & G9 V \\
\hline HD $37572 \ldots \ldots \ldots$ & 053656.86 & -475752.87 & K0 V \\
\hline HD 37216 & 053952.33 & +525350.83 & G5 \\
\hline HD 37962 & 054051.97 & -312103.95 & G5 V \\
\hline HD $37006 \ldots \ldots \ldots$ & 054611.89 & +78 1522.61 & G0 \\
\hline HD 38529 & 054634.92 & +01 1005.31 & G8 III/IV \\
\hline HD $38949 \ldots$ & 054820.06 & -242750.04 & G1 V \\
\hline HD $40647 \ldots \ldots .$. & 060605.68 & +692834.02 & G5 \\
\hline HD $43989 \ldots \ldots \ldots$ & 061908.05 & -032620.39 & G0 V \\
\hline HD $44594^{\mathrm{b}}$... & 062006.16 & -484428.05 & G3 V \\
\hline HD $45270 \ldots$ & 062230.97 & $\begin{array}{llll}-60 & 13 & 07.14\end{array}$ & G1 V \\
\hline HD $61005^{\mathrm{a}}$ & 073547.47 & -321214.11 & $\mathrm{G} 3 / 5 \mathrm{~V}$ \\
\hline HD 60737 & 073816.44 & +47 4455.34 & G0 \\
\hline HD $61994 \ldots \ldots \ldots$ & 074730.61 & +70 1223.97 & G6 V \\
\hline HD $64324 \ldots \ldots$. & 075448.47 & +34 3711.42 & G0 \\
\hline HD $66751 \ldots \ldots$. & 081020.51 & +694330.21 & F8 V \\
\hline HD $69076 \ldots \ldots \ldots$ & $08 \quad 1507.73$ & -065508.23 & K0 V \\
\hline HD $70516 \ldots \ldots$ & 082415.66 & +445658.92 & G0 \\
\hline HD $71974 \ldots \ldots \ldots$ & 083135.05 & +34 5758.44 & G5 \\
\hline HD $72687 \ldots \ldots \ldots$ & $\begin{array}{lll}08 & 33 & 15.39\end{array}$ & -295723.66 & G5 V \\
\hline HD $73668 \ldots \ldots \ldots$ & 083943.81 & +054551.59 & G1 V \\
\hline HIP $42491 \ldots \ldots \ldots$ & 083944.69 & +05 4614.00 & G5 \\
\hline HD $75302 \ldots \ldots \ldots$ & $\begin{array}{llll}08 & 49 & 12.53\end{array}$ & +032905.25 & G5 V \\
\hline HD 75393 & $0849 \quad 15.35$ & -153353.12 & F7 V \\
\hline HD $76218 \ldots \ldots \ldots$ & 085555.68 & +36 1146.40 & G9 V \\
\hline HD $77407 \ldots \ldots$. & 090327.08 & +3750 27.72 & G0 (V) \\
\hline HD $80606 \ldots \ldots \ldots$ & 092237.56 & +50 3613.43 & G5 \\
\hline HD $85301 \ldots \ldots \ldots$ & 095216.77 & +49 1126.84 & G5 \\
\hline HD $88201 \ldots \ldots$. & 100931.78 & -325047.95 & G0 V \\
\hline HD $88742 \ldots \ldots \ldots$ & 101324.72 & -330154.22 & G0 V \\
\hline HD 90712 & 102747.79 & -342358.14 & $\mathrm{G} 2 / 3 \mathrm{~V}$ \\
\hline HD $90905 \ldots \ldots$. & 102942.23 & +012927.82 & G1 V \\
\hline HD $91782 \ldots \ldots \ldots$ & 103647.84 & +47 4312.42 & G0 \\
\hline HD $91962 \ldots \ldots \ldots$ & 103700.02 & -085023.63 & G1 V \\
\hline HD $92788 \ldots \ldots \ldots$ & 104248.54 & -021101.38 & G6 V \\
\hline HD $92855 \ldots \ldots \ldots$ & 104400.62 & +46 1223.86 & F9 V \\
\hline HD $95188 \ldots \ldots \ldots$ & 105948.28 & +251723.65 & G8 V \\
\hline HD $98553 \ldots \ldots$. & 112011.60 & -193440.54 & $\mathrm{G} 2 / 3 \mathrm{~V}$ \\
\hline HD $100167 \ldots \ldots$ & 113153.92 & +412621.65 & F8 \\
\hline HD $101472 \ldots \ldots$ & 114036.59 & -082420.32 & F7 V \\
\hline
\end{tabular}


TABLE 1 (Continued)

\begin{tabular}{|c|c|c|c|}
\hline Name & R.A. (J2000.0) & Decl. (J2000.0) & Spectral Type \\
\hline HD 101959 & 114356.62 & -294451.80 & G0 V \\
\hline HD $102071 \ldots \ldots$ & 114439.32 & -295305.46 & $\mathrm{~K} 0 \mathrm{~V}$ \\
\hline HD $103432 \ldots \ldots$ & 115432.07 & +192440.44 & G6 V \\
\hline HD $104576 \ldots$ & 120239.46 & -104249.16 & G3 V \\
\hline HD $104860 \ldots \ldots$ & 120433.71 & +662011.58 & F8 \\
\hline HD $105631 \ldots \ldots$ & 120937.26 & +401507.62 & G9 V \\
\hline HD $106156 \ldots \ldots$ & 121257.52 & +100215.62 & G8 V \\
\hline HD $106252 \ldots \ldots$ & 121329.49 & +100229.96 & G0 \\
\hline HD $107146 \ldots \ldots$ & 121906.49 & +163253.91 & $\mathrm{G} 2 \mathrm{~V}$ \\
\hline HD $108799 \ldots \ldots$ & 123004.77 & -132335.14 & $\mathrm{G} 1 / 2 \mathrm{~V}$ \\
\hline HD 108944 & 123100.74 & +312525.84 & F9 V \\
\hline HD 112196 & 125440.02 & +220628.65 & F8 V \\
\hline HD $115043 \ldots \ldots$ & 131337.01 & +564229.82 & G1 V \\
\hline HD $121320 \ldots \ldots$ & 135428.20 & +203830.46 & G5 V \\
\hline HD $121504 \ldots \ldots \ldots$ & 135717.23 & -560224.27 & $\mathrm{G} 2 \mathrm{~V}$ \\
\hline HD $122652 \ldots \ldots$. & 140231.63 & +313939.09 & F8 \\
\hline HD $129333 \ldots \ldots$ & 143900.25 & +641729.94 & G5 V \\
\hline HD $132173 \ldots \ldots$ & 145830.51 & -284234.15 & G0 V \\
\hline HD $133295 \ldots \ldots$ & 150433.08 & -281800.65 & $\mathrm{G} 0 / 1 \mathrm{~V}$ \\
\hline HD 136923 & 152246.84 & +185508.31 & G9 V \\
\hline HD $138004 \ldots \ldots \ldots$ & 152740.36 & +425252.82 & G2 III \\
\hline HD $139813 \ldots \ldots$ & 152923.61 & +802701.08 & G5 \\
\hline HD $141937 \ldots \ldots$ & 155217.55 & -182609.80 & $\mathrm{G} 2 / 3 \mathrm{~V}$ \\
\hline HD 142229 & 155320.02 & +041511.51 & G5 V \\
\hline HD 145229 & 160926.63 & +113428.25 & G0 \\
\hline HD $150706 \ldots \ldots$ & 163117.63 & +794723.15 & G3 (V) \\
\hline HD $150554 \ldots \ldots$ & 164056.45 & +215653.24 & F8 \\
\hline HD $151798 \ldots \ldots$ & 165005.17 & -122314.88 & G3 V \\
\hline HD $152555 \ldots \ldots$ & 165408.15 & -042024.89 & F8/G0 V \\
\hline HD $153458 \ldots \ldots$ & 170001.66 & -073153.93 & G5 V \\
\hline HD 154417 & 170516.83 & +004209.18 & F9 V \\
\hline HD $157664^{\mathrm{b}}$ & 171858.47 & +685240.61 & G0 \\
\hline HD $159222 \ldots \ldots$ & 173200.99 & +341615.97 & G1 V \\
\hline HD 161897 & 174106.70 & +722513.41 & K0 \\
\hline HD 167389 & 181307.22 & +412831.33 & F8 (V) \\
\hline HD $170778 \ldots \ldots$ & 182903.94 & +435621.54 & G5 \\
\hline HD $172649 \ldots \ldots$ & 183942.11 & +375935.22 & F5 \\
\hline HD $179949 \ldots \ldots$ & 191533.23 & $-24 \quad 1045.61$ & F8 V \\
\hline HD 183216 & 192940.57 & -304752.36 & G2 V \\
\hline HD $187897 \ldots \ldots$ & 195209.38 & +072736.10 & G5 \\
\hline HD $190228 \ldots \ldots$. & 200300.77 & +281824.46 & G5 IV \\
\hline HD 193017 & 201810.00 & -044343.23 & F6 V \\
\hline HD 195034 & 202811.81 & +220744.34 & G5 \\
\hline HD $199019 \ldots \ldots$ & 204929.30 & +714629.29 & G5 \\
\hline HD $199598 \ldots \ldots$. & 205739.68 & +262418.40 & G0 V \\
\hline HD $200746 \ldots \ldots$. & 210507.95 & +075643.59 & G5 \\
\hline HD $201219 \ldots \ldots$ & 210756.53 & +072558.47 & G5 \\
\hline HD $202108 \ldots \ldots \ldots$ & 211257.63 & +304834.25 & G3 V \\
\hline HD $201989 \ldots \ldots$ & 211401.80 & -293948.85 & $\mathrm{G} 3 / 5 \mathrm{~V}$ \\
\hline HD $203030 \ldots$ & 211858.22 & +261350.05 & G8 V \\
\hline HD $204277 \ldots \ldots$ & 212706.61 & +1607 26.85 & F8 V \\
\hline HD $205905 \ldots \ldots$ & 213910.14 & -271823.59 & G2 V \\
\hline HD $206374 \ldots \ldots$. & 214106.19 & +264502.25 & G6.5 V \\
\hline HD $209393 \ldots \ldots$. & 220205.38 & +442035.47 & G5 \\
\hline HD $209779 \ldots \ldots$ & 220605.32 & -052129.15 & G2 V \\
\hline HD $212291 \ldots \ldots$ & 222309.17 & +09 2739.95 & G5 \\
\hline HD $216275 \ldots \ldots$ & 225046.34 & +520341.21 & G0 \\
\hline HD $217343 \ldots \ldots$ & 230019.29 & -260913.48 & G3 V \\
\hline
\end{tabular}

Note.-Units of right ascension are hours, minutes, and seconds, and units of declination are degrees, arcminutes, and arcseconds.

a IRS high-resolution spectra were also obtained for these stars.

b These stars are also Spitzer Space Telescope calibration targets. 
TABLE 2

Open Cluster Stars

\begin{tabular}{|c|c|c|c|c|}
\hline Name & R.A. (J2000.0) & Decl. (J2000.0) & Spectral Type & Open Cluster \\
\hline vB 1 & $\begin{array}{lll}03 & 1726.39\end{array}$ & +073920.90 & F8 & Hyades \\
\hline HE 350 & 031736.93 & +485008.50 & $\ldots$ & $\alpha$ Per \\
\hline HE 373 & $03 \quad 1827.39$ & +472115.42 & $\ldots$ & $\alpha$ Per \\
\hline HE 389 & $03 \quad 1850.31$ & +494352.19 & $\ldots$ & $\alpha$ Per \\
\hline AP $93 \ldots \ldots \ldots$ & 031902.76 & +48 1059.61 & $\ldots$ & $\alpha$ Per \\
\hline HE $622 \ldots \ldots$ & 032449.71 & +485218.33 & $\ldots$ & $\alpha$ Per \\
\hline HE 696 & $03 \quad 2619.36$ & +49 1332.54 & $\ldots$ & $\alpha$ Per \\
\hline HE 699 & $\begin{array}{lll}03 & 26 & 22.22\end{array}$ & +492537.52 & $\ldots$ & $\alpha$ Per \\
\hline HE $750 \ldots \ldots$ & $03 \quad 2737.79$ & +485928.78 & F5 & $\alpha$ Per \\
\hline HE 767 & 032755.02 & +494537.16 & $\ldots$ & $\alpha$ Per \\
\hline HE 848 & 032926.24 & $+48 \quad 1211.74$ & F9 V & $\alpha$ Per \\
\hline HE 935 & 033128.99 & +485928.37 & F9.5 V & $\alpha$ Per \\
\hline HE $1101 \ldots \ldots$ & 033508.75 & +494439.59 & $\ldots$ & $\alpha$ Per \\
\hline HE $1234 \ldots \ldots$ & 033902.91 & +513637.11 & $\ldots$ & $\alpha$ Per \\
\hline HII $120 \ldots \ldots$ & 034331.95 & +234026.61 & $\ldots$ & Pleiades \\
\hline HII $152 \ldots \ldots$ & $03 \quad 43 \quad 37.73$ & +233209.59 & G5 V & Pleiades \\
\hline HII $174 \ldots \ldots$. & 034348.33 & +250015.83 & $\ldots$ & Pleiades \\
\hline HII $173 \ldots \ldots$ & 034348.41 & +251124.19 & $\ldots$ & Pleiades \\
\hline HII $250 \ldots \ldots$ & $03 \quad 4404.24$ & +245923.40 & $\ldots$ & Pleiades \\
\hline HII $314 \ldots \ldots$ & 034420.09 & +244746.16 & $\cdots$ & Pleiades \\
\hline HII $514 \ldots \ldots$ & 034504.01 & +251528.23 & $\ldots$ & Pleiades \\
\hline HII $1015 \ldots \ldots$ & 034627.35 & +250807.97 & $\ldots$ & Pleiades \\
\hline HII $1101 \ldots \ldots$ & 034638.78 & +245734.61 & G0 V & Pleiades \\
\hline HII $1182 \ldots \ldots$ & 034647.06 & +225452.48 & $\ldots$ & Pleiades \\
\hline HII $1200 \ldots \ldots$ & 034650.54 & +231421.06 & $\ldots$ & Pleiades \\
\hline HII $1776 \ldots \ldots$ & $03 \quad 48 \quad 17.70$ & +250252.29 & $\ldots$ & Pleiades \\
\hline HII $2147 \ldots \ldots$ & 034906.11 & +234652.49 & G7 IV & Pleiades \\
\hline HII $2278 \ldots \ldots$ & $03 \quad 4925.70$ & +245615.43 & $\ldots$ & Pleiades \\
\hline HII $2506 \ldots \ldots$ & 034956.49 & +231307.01 & $\ldots$ & Pleiades \\
\hline HII $2644 \ldots . .$. & 035020.90 & +242800.22 & $\ldots$ & Pleiades \\
\hline HII $2786 \ldots \ldots$ & 035040.08 & +235558.94 & $\ldots$ & Pleiades \\
\hline HII $2881 \ldots \ldots$ & 035054.32 & +235005.52 & $\mathrm{~K} 2$ & Pleiades \\
\hline HII $3097 \ldots . .$. & 035140.44 & +245859.41 & $\ldots$ & Pleiades \\
\hline HII $3179 \ldots \ldots$ & 035156.86 & +235406.98 & $\ldots$ & Pleiades \\
\hline vB $39 \ldots \ldots \ldots$ & 042244.74 & +164727.56 & G4 V & Hyades \\
\hline vB $49 \ldots \ldots \ldots$ & 042412.78 & +162244.22 & G0 V & Hyades \\
\hline vB 52 & 042428.33 & +165310.32 & $\mathrm{G} 2 \mathrm{~V}$ & Hyades \\
\hline vB $176 \ldots \ldots \ldots$ & 042547.56 & +180102.20 & $\mathrm{K} 2 \mathrm{~V}$ & Hyades \\
\hline vB $63 \ldots \ldots \ldots$ & 042624.61 & +165111.84 & G1 V & Hyades \\
\hline vB $64 \ldots \ldots \ldots$ & 042640.11 & +164448.78 & $\mathrm{G} 2+$ & Hyades \\
\hline vB $66 \ldots \ldots \ldots$ & 042746.07 & +114411.07 & F8 & Hyades \\
\hline vB $73 \ldots \ldots \ldots$ & 042848.29 & +171707.84 & $\mathrm{G} 2 \mathrm{~V}$ & Hyades \\
\hline vB $79 \ldots \ldots \ldots$ & 042931.61 & +175335.46 & $\mathrm{~K} 0 \mathrm{~V}$ & Hyades \\
\hline vB $180 \ldots \ldots \ldots$ & 042957.73 & +164022.23 & $\mathrm{~K} 1 \mathrm{~V}$ & Hyades \\
\hline vB $88 \ldots \ldots \ldots$ & $\begin{array}{lll}04 & 31 & 29.35\end{array}$ & +135412.55 & F9 V & Hyades \\
\hline vB $91 \ldots \ldots \ldots$ & 043250.12 & +160020.96 & $\ldots$ & Hyades \\
\hline vB $92 \ldots \ldots \ldots$ & 043259.45 & +154908.37 & $\ldots$ & Hyades \\
\hline vB $93 \ldots \ldots \ldots$ & 043337.97 & +164544.96 & $\ldots$ & Hyades \\
\hline vB $96 \ldots \ldots \ldots$ & 043358.54 & +150949.04 & G5 & Hyades \\
\hline vB $183 \ldots \ldots \ldots$ & 043432.18 & +154939.23 & $\ldots$ & Hyades \\
\hline vB $97 \ldots \ldots \ldots$ & 043435.31 & +153016.56 & F8 V & Hyades \\
\hline vB $99 \ldots \ldots \ldots$ & 043605.27 & +154102.60 & $\ldots$ & Hyades \\
\hline vB $106 \ldots \ldots \ldots$ & 043857.31 & +140620.16 & G5 & Hyades \\
\hline vB $142 \ldots \ldots \ldots$ & 044630.38 & +152819.38 & G5 & Hyades \\
\hline vB $143 \ldots \ldots$. & 045123.22 & +152600.45 & F8 & Hyades \\
\hline R3 $\ldots \ldots \ldots \ldots$ & 102932.75 & -634915.68 & $\ldots$ & IC 2602 \\
\hline $\mathrm{R} 45 \ldots \ldots \ldots \ldots$ & 104000.03 & -631511.04 & $\cdots$ & IC 2602 \\
\hline ......... & 104207.07 & -644607.85 & $\ldots$ & IC 2602 \\
\hline B102 ........... & 104241.52 & -642104.37 & $\ldots$ & IC 2602 \\
\hline R83 $\ldots \ldots \ldots \ldots$ & 104614.83 & -640258.05 & $\ldots$ & IC 2602 \\
\hline
\end{tabular}


TABLE 3

Young Stars

\begin{tabular}{|c|c|c|c|}
\hline Name & R.A. (J2000.0) & Decl. (J2000.0) & Spectral Typ \\
\hline $\mathrm{HD} 105^{\mathrm{a}}$ & 000552.56 & -414510.98 & G0 V \\
\hline QT And & $0041 \quad 17.32$ & +34 2516.77 & G \\
\hline RE J0137+18A & 013739.41 & +183533.16 & $\mathrm{~K} 3 \mathrm{Ve}$ \\
\hline HD 15526 & 022935.03 & -122408.56 & $\mathrm{G} 5 / 6 \mathrm{~V}$ \\
\hline 1RXS J025216.9+361658 ....... & $0252 \quad 17.59$ & +36 1648.14 & K2 IV \\
\hline 2RE J0255+474 _............... & 025543.60 & +474647.58 & $\mathrm{~K} 5 \mathrm{Ve}$ \\
\hline 1RXS J025751.8+115759 & 025751.68 & +115805.83 & G7 V \\
\hline RX J0258.4+2947 ........ & 025828.77 & +294753.80 & K0 IV \\
\hline 1RXS J030759.1+302032 & 030759.20 & +302026.05 & G5 IV \\
\hline 1E $0307.4+1424$ & $\begin{array}{lll}03 & 10 & 12.55\end{array}$ & +143602.90 & G6 V \\
\hline 1RXS J031644.0+192259 & 031643.89 & +192304.11 & $\mathrm{G} 2 \mathrm{~V}$ \\
\hline 1RXS J031907.4+393418 & 031907.61 & +3934 10.50 & $\mathrm{~K} 0 \mathrm{~V}$ \\
\hline 1E $0324.1-2012$ & 032622.05 & -200148.81 & G4 V \\
\hline RX J0329.1+0118 & 032908.06 & +01 1805.66 & G0 (IV) \\
\hline RX J0331.1+0713 & 033108.38 & +071324.78 & $\mathrm{~K} 4(\mathrm{~V}) / \mathrm{E}$ \\
\hline HD $22179 \ldots \ldots \ldots$ & 033529.91 & +311337.45 & G5 IV \\
\hline 1RXS J034423.3+281224. & 034424.25 & +281223.07 & G7 V \\
\hline 1RXS J035028.0+163121 ....... & 035028.40 & +163115.19 & G5 IV \\
\hline RX J0354.4+0535 ....... & 035421.31 & +053540.77 & $\mathrm{G} 2(\mathrm{~V})$ \\
\hline RX J0357.3+1258 & 035721.39 & +125816.83 & G0 \\
\hline HD $25300 \ldots \ldots \ldots$ & 035936.73 & -395314.85 & K0 \\
\hline HD 285281 & 040031.07 & +193520.70 & $\mathrm{K} 1$ \\
\hline HD 285372 & 040324.95 & +172426.12 & K3 (V) \\
\hline HD $284135^{\mathrm{a}}$ & 040540.58 & +224812.14 & G3 (V) \\
\hline HD 281691 & 040909.74 & +290130.55 & $\mathrm{K} 1(\mathrm{~V})$ \\
\hline HD $26182 \ldots$ & 041004.69 & +36 3912.14 & G0 V \\
\hline HD 284266 & 041522.92 & +204416.93 & $\mathrm{~K} 0(\mathrm{~V})$ \\
\hline HD 285751 & 042341.33 & +153754.87 & $\mathrm{~K} 2(\mathrm{~V})$ \\
\hline HD 279788 & 042637.40 & +384502.37 & G5 V \\
\hline HD 285840 & 043242.43 & +18 5510.25 & $\mathrm{~K} 1(\mathrm{~V})$ \\
\hline 1RXS J043243.2-152003 & 043243.51 & -152011.39 & $\mathrm{G} 4 \mathrm{~V}$ \\
\hline RX J0434.3+0226 ....... & 043419.54 & +022626.10 & $\mathrm{~K} 4 \mathrm{e}$ \\
\hline HD 282346 & 043931.00 & +340744.43 & G8 V \\
\hline RX J0442.5+0906 & 044232.09 & +090600.86 & G5 $(\mathrm{V})$ \\
\hline HD $31281 \ldots \ldots \ldots$ & 045509.62 & +182630.84 & G1 (V) \\
\hline HD 286179 & 045700.65 & +15 1753.09 & G3 (V) \\
\hline HD 31950 . & 050024.31 & +150525.28 & $\ldots$ \\
\hline HD 286264 & $0500 \quad 49.28$ & +152700.68 & $\mathrm{K} 2 \mathrm{IV}$ \\
\hline 1RXS J051111.1+281353 & $\begin{array}{lll}05 & 11 & 10.53\end{array}$ & +281350.38 & $\mathrm{~K} 0 \mathrm{~V}$ \\
\hline 1RXS J053650.0+133756 & 053650.06 & +13 3756.22 & $\mathrm{~K} 0 \mathrm{~V}$ \\
\hline HD 245567 & 053718.44 & +133452.52 & G0 V \\
\hline SAO 150676 & $05 \quad 4020.74$ & -194010.85 & $\mathrm{G} 2 \mathrm{~V}$ \\
\hline AO Men ${ }^{\mathrm{a}}$ & $06 \quad 18 \quad 28.24$ & -720241.56 & $\mathrm{~K} 3.5$ \\
\hline HD 47875 & 063441.04 & -695306.35 & G3 V \\
\hline RE J0723+20 & 072343.58 & +202458.64 & $\mathrm{K} 3(\mathrm{~V})$ \\
\hline HD $70573 \ldots$ & 082249.95 & +015133.58 & $\mathrm{G} 1 / 2 \mathrm{~V}$ \\
\hline RX J0849.2-7735 & 084911.11 & -773558.53 & $\mathrm{~K} 1(\mathrm{~V})$ \\
\hline RX J0850.1-7554 & 085005.41 & -755438.11 & G5 \\
\hline RX J0853.1-8244 & 085305.29 & -824359.71 & $\mathrm{~K} 0(\mathrm{~V})$ \\
\hline RX J0917.2-7744 & $09 \quad 17 \quad 10.33$ & -774401.99 & $\mathrm{G} 2$ \\
\hline HD 86356 & 095150.70 & -790137.73 & G6/K0 \\
\hline SAO 178272 & 095908.42 & -223934.57 & $\mathrm{~K} 2 \mathrm{~V}$ \\
\hline MML $1 \ldots \ldots \ldots \ldots$ & 105749.37 & -691359.99 & K1 IV \\
\hline RX J1111.7-7620 & 111146.32 & -762009.21 & K1 \\
\hline RX J1140.3-8321. & 114016.59 & $\begin{array}{lll}-8321 & 00.38\end{array}$ & K2 \\
\hline BPM $87617 \ldots \ldots \ldots \ldots$ & 114745.73 & +125403.31 & $\mathrm{K} 5 \mathrm{Ve}$ \\
\hline HD 104467 & 120139.15 & -785916.85 & G5 III/IV \\
\hline RX J1203.7-8129 & 120324.70 & -812955.28 & K1 \\
\hline HIP 59154 & 120751.19 & -755515.97 & $\mathrm{~K} 2$ \\
\hline RX J1209.8-7344 & 120942.82 & -734441.41 & G9 \\
\hline MML $8^{\mathrm{a}}$ & 121235.77 & -552027.31 & K0 IV \\
\hline MML 9. & 121434.10 & $\begin{array}{llll}-51 & 10 & 12.47\end{array}$ & G9 IV \\
\hline
\end{tabular}


TABLE 3 (Continued)

\begin{tabular}{|c|c|c|c|}
\hline Name & R.A. (J2000.0) & Decl. (J2000.0) & Spectral Type \\
\hline HD 106772 & 121726.94 & -803506.90 & G2 III/IV \\
\hline RX J1220.6-7539 & 122034.38 & -753928.65 & $\mathrm{~K} 2$ \\
\hline HD 107441 & 122116.48 & -531745.06 & G1.5 IV \\
\hline MML $17^{\mathrm{a}}$ & 122233.23 & -533348.95 & G0 IV \\
\hline MML 18 & 122340.13 & -561632.57 & K0 IV \\
\hline RX J1225.3-7857 & 122513.40 & -785734.71 & G5 \\
\hline HD 111170 & 124751.86 & -512638.29 & G8/K0 V \\
\hline MML 26 & 124848.19 & -563537.90 & G5 IV \\
\hline MML $28^{\mathrm{a}}$. & 130150.70 & -530458.11 & K2 IV \\
\hline MML 32 & 131756.94 & -531756.21 & G1 IV \\
\hline HD 116099 & 132204.47 & -450323.19 & $\mathrm{G} 0 / 3$ \\
\hline PDS $66^{\mathrm{a}}$ & 132207.53 & -693812.18 & K1 IVe \\
\hline HD 117524 & 133153.61 & $\begin{array}{lll}-51 & 13 & 33.05\end{array}$ & G2.5 IV \\
\hline MML $36^{\mathrm{a}} .$. & 133757.30 & -413441.98 & K0 IV \\
\hline HD 119269 & 134328.54 & -543643.44 & $\mathrm{G} 3 / 5 \mathrm{~V}$ \\
\hline MML 38 & 134750.55 & -490205.61 & G8 IVe \\
\hline HD 120812 & 135247.80 & -464409.24 & F8/G0 V \\
\hline MML $40 \ldots$ & 140220.72 & -414450.93 & G9 IV \\
\hline MML 43 & 142705.56 & -471421.73 & G7 IV \\
\hline HD 126670 & 142809.30 & -441417.54 & G6/8 III/IV \\
\hline HD 128242 & 143704.22 & -414502.91 & G3 V \\
\hline RX J1450.4-3507 & 145025.82 & -350648.66 & K1 (IV) \\
\hline MML $51 \ldots \ldots \ldots$ & 145241.98 & -414155.24 & $\mathrm{~K} 1 \mathrm{IVe}$ \\
\hline RX J1457.3-3613 ... & 145719.62 & $-36 \quad 1227.44$ & G6 IV \\
\hline RX J1458.6-3541 ….......... & 145837.69 & -354030.27 & K3 (IV) \\
\hline RX J1500.8-4331 f........... & 150051.89 & -433121.23 & K1 (IV) \\
\hline MML $57 \ldots$ & 150158.82 & -475546.46 & G1.5 IV \\
\hline RX J1507.2-3505 & 150714.81 & -350459.55 & K0 \\
\hline HD $135363 \ldots \ldots$ & 150756.31 & +761202.66 & G5 (V) \\
\hline HD 133938 & 150838.50 & -440051.99 & G6/8 III/IV \\
\hline RX J1518.4-3738 & 151826.92 & -373802.14 & K1 \\
\hline RX J1531.3-3329 & 153121.93 & -332939.46 & K0 \\
\hline HIP $76477 \ldots$ & 153711.30 & -401556.70 & G9 \\
\hline V343 Nor $^{\mathrm{a}}$ & 153857.57 & -574227.30 & $\mathrm{~K} 0 \mathrm{~V}$ \\
\hline HD 139498 & 153924.40 & -271021.87 & G8 (V) \\
\hline RX J1541.1-2656 ............. & 154106.79 & -265626.33 & G7 \\
\hline RX J1544.0-3311 .............. & 154403.76 & -331111.09 & K1 \\
\hline HD $140374 \ldots \ldots \ldots \ldots \ldots \ldots$ & 154421.06 & -331854.97 & G8 V \\
\hline RX J1545.9-4222 ............. & 154552.25 & -422216.41 & K1 \\
\hline HD 141521 & 155113.74 & -421851.36 & G8 V \\
\hline HD $141943^{a}$ & 155327.29 & -421600.81 & $\mathrm{G} 0 / 2 \mathrm{~V}$ \\
\hline HD $142361^{a}$ & 155459.86 & -234718.26 & G3 V \\
\hline [PZ99] J155847.8-175800 ...... & 155847.73 & -175759.58 & K3 \\
\hline RX J1600.6-2159 a $\ldots \ldots$ & 160040.57 & -220032.24 & G9 \\
\hline HD $143358 \ldots$ & 160107.93 & -325452.65 & $\mathrm{G} 1 / 2 \mathrm{~V}$ \\
\hline Sco PMS 21 & 160125.63 & -224040.38 & K1 IV \\
\hline Sco PMS $27 \ldots \ldots \ldots \ldots \ldots \ldots$ & 160447.76 & -193023.12 & K2 IV \\
\hline [PZ99] J160814.7-190833 .......... & 160814.74 & -190832.77 & $\mathrm{~K} 2$ \\
\hline Sco PMS $52^{\mathrm{a}}$ & 161240.51 & -185928.31 & K0 IV \\
\hline [PZ99] J161318.6-221248 ....... & 161318.59 & -221248.96 & G9 \\
\hline [PZ99] J161329.3-231106 ...... & 161329.29 & -231107.56 & $\mathrm{~K} 1$ \\
\hline [PZ99] J161402.1-230101 ....... & 161402.12 & -230102.18 & G4 \\
\hline [PZ99] J161411.0-230536 $\ldots$.... & 161411.08 & -230536.26 & K0 \\
\hline [PZ99] J161459.2-275023 & 161459.18 & -275023.06 & G5 \\
\hline [PZ99] J161618.0-233947. & 161617.95 & -233947.70 & G7 \\
\hline HD $146516 \ldots \ldots$ & 161731.39 & -230336.02 & G0 IV \\
\hline Sco PMS $214^{\mathrm{a}}$ & 162948.70 & -215211.91 & K0 IV \\
\hline RX J1839.0-3726 & 183905.29 & -372621.78 & $\mathrm{~K} 1$ \\
\hline RX J1841.8-3525 ... & 184148.56 & -352543.71 & G7 \\
\hline RX J1842.9-3532 & 184257.98 & -353242.73 & $\mathrm{~K} 2$ \\
\hline RX J1844.3-3541 ... & 184421.92 & -354143.53 & K5 \\
\hline RX J1852.3-3700 & $1852 \quad 17.30$ & -370011.93 & K3 \\
\hline
\end{tabular}


TABLE 3 (Continued)

\begin{tabular}{lcccc}
\hline \hline \multicolumn{1}{c}{ Name } & R.A. (J2000.0) & Decl. (J2000.0) & Spectral Type \\
\hline HD 174656 $\ldots \ldots \ldots \ldots \ldots \ldots \ldots \ldots$ & 185305.99 & -361022.91 & G6 IV \\
RX J1917.4 & $-3756^{\mathrm{a}} \ldots \ldots \ldots \ldots \ldots \ldots$ & 191723.83 & -375650.52 & K2 \\
HD 199143 & $\ldots \ldots \ldots \ldots \ldots \ldots \ldots \ldots \ldots$ & 205547.68 & -170651.02 & F8 V \\
V383 Lac $\ldots \ldots \ldots \ldots \ldots \ldots \ldots \ldots \ldots$ & 222007.03 & +493011.67 & K0 IV/V \\
RX J2313.0 $2345 \ldots \ldots \ldots \ldots \ldots \ldots$ & 231301.24 & +234529.64 & F8 \\
HD 219498 $\ldots \ldots \ldots \ldots \ldots \ldots \ldots \ldots$ & 231605.02 & +221034.98 & G5 \\
\hline
\end{tabular}

${ }^{a}$ IRS high-resolution spectra were also obtained for these stars.

stars also observed at $160 \mu \mathrm{m}$ ), including 7-38 $\mu \mathrm{m}$ low-resolution IRS spectra. A detailed description of the observing commands (Astronomical Observing Requests [AORs]) that specify the FEPS program can be found using the Spitzer Science Center's (SSC's) SPOT software to "View Program" and entering program identification number (PID) 148. In this section, we outline the AOR strategy for each instrument.

Data are obtained with IRAC in three bands $(3.6,4.5$, and $8.0 \mu \mathrm{m})$. The first five FEPS objects observed as part of the early verification program were observed in all four IRAC bands (including the $5.6 \mu \mathrm{m}$ channel). FEPS stars are observed in the IRAC subarray mode $(32 \times 32$ pixels $)$ at frame times of $0.02,0.1$, or $0.4 \mathrm{~s}$, using the " 4 point random" dither pattern at the medium dither scale. At each of the four dither positions, 64 images are taken at the same frame time in each band. Thus, there are 256 images of each star for each IRAC band.

Low-resolution $(\mathcal{R} \approx 64-128)$ spectra covering the longer wavelength ranges (7.4-38 $\mu \mathrm{m})$ in the SL1, LL1, and LL2 modules of the IRS are obtained for all FEPS objects. Observations using the SL2 module (5.2-7.7 $\mu \mathrm{m})$ were also obtained for the five validation stars and for objects younger than $30 \mathrm{Myr}$. Highresolution $(\mathcal{R} \approx 600)$ spectra are obtained for the 35 stars chosen for the gas-detection experiment. All IRS observations use standard starring mode and high-accuracy peak-up.

MIPS photometric imaging data at 24 and $70 \mu \mathrm{m}$ are obtained

TABLE 4

Preselected IRS High-Resolution Targets

\begin{tabular}{|c|c|c|c|}
\hline Name & R.A. (J2000.0) & Decl. (J2000.0) & Spectral Type \\
\hline HD 8907 & 012834.35 & +421603.70 & F8 \\
\hline HD 17925 & 025232.14 & -124611.18 & $\mathrm{~K} 1 \mathrm{~V}$ \\
\hline HD 25457 & 040236.76 & -001608.17 & F7 V \\
\hline HD 35850 & 052704.77 & -115403.38 & $\mathrm{~F} 7 / 8 \mathrm{~V}$ \\
\hline HD 37484 & 053739.63 & -283734.65 & F3 V \\
\hline HD $38207^{a}$ & 054320.95 & -201121.41 & $\mathrm{~F} 2 \mathrm{~V}$ \\
\hline HD $41700 \ldots$ & 060428.44 & -450211.71 & F8/G0 V \\
\hline HD $72905^{\mathrm{a}} \ldots$ & 083911.62 & +650115.14 & G1.5 VB \\
\hline HD 134319 . & 150549.90 & +640250.00 & G5 (V) \\
\hline HD $143006 \ldots \ldots$. & 155836.92 & -225715.35 & G6/8 \\
\hline HD $191089^{\mathrm{a}}$. & 200905.22 & -261326.63 & F5 V \\
\hline HD $202917 \ldots \ldots$ & 212049.95 & $\begin{array}{lll}-53 & 02 & 03.05\end{array}$ & G5 V \\
\hline HD $209253 \ldots \ldots$ & 220232.97 & -320801.60 & F6/7 V \\
\hline HD $216803 \ldots \ldots$ & 225624.07 & -313356.12 & K4 VP \\
\hline
\end{tabular}

${ }^{a}$ IRS high-resolution spectra were not obtained for these sources, due to program constraints. for the entire FEPS sample. We achieve S/N $>30$ at $\leq 24 \mu \mathrm{m}$ for our entire sample and $\mathrm{S} / \mathrm{N}>5$ at the photospheric limit for as much of our sample as is practical at $70 \mu \mathrm{m}$. For sources whose photospheres we are unable to detect in a reasonable time (10 cycles), we achieve $\mathrm{S} / \mathrm{N}>5$ on dust debris at 5 times the current solar system values. MIPS $160 \mu \mathrm{m}$ data are also obtained for $10 \%$ of the lowest background targets, chosen to span the age range of the full sample. The MIPS default scale photometric mode acquires data in multiple pointings, with small offsets between each pointing to alleviate instrumental artifacts and cosmic rays. Due to the stability of the $\mathrm{Si}$ : As array, these multiple pointings for the $24 \mu \mathrm{m}$ data allow repeatability to be used as an accurate estimate of internal measurement (precision) uncertainty. For 70 and $160 \mu \mathrm{m}$, the multiple pointings are used to calibrate the time-dependent detector response, and therefore only a final mosaic image is produced by the photometry mode for the Ge : Ga arrays.

\subsubsection{Data Reduction}

The SSC pipelined data products from FEPS can be accessed through the SSC's Leopard archive browser. In this section, we outline the general data reduction strategy for each instrument. We refer the reader to FEPS data publications for a more detailed description of the data reduction applied to individual sources (see also the FEPS Explanatory Supplements that accompany our data releases through the SSC).

\subsubsection{IRAC}

IRAC data frames are processed through the SSC pipeline to produce basic calibrated data (BCD) images. Sixty-four images are obtained at each of the four dither positions, for a total of 256 images of the object in each band.

Flux densities are derived using aperture photometry. A Gaussian fit to the point-spread function (PSF) is used to center the target aperture. The target and background annuli are optimized for the ensemble of observations to maximize the measured $\mathrm{S} / \mathrm{N}$. These measured flux densities are then corrected to the standard calibration aperture sizes supplied by the SSC. To estimate measurement uncertainties, we assign the standard deviation of the 256 measurements as the $1 \sigma$ internal uncertainty. The total uncertainty reported by FEPS is the (square) root of the sum of squares (RSS) of this internal measurement uncer- 


\section{MEYER ET AL.}

tainty and the calibration uncertainty as published by the SSC. Although the calibration of IRAC assumes a flat $\left(\nu F_{\nu}=\right.$ const.) spectrum across the bandpass, no color corrections have been applied to the FEPS IRAC measurements. The prescription for color corrections is presented in the IRAC Data Handbook available through the SSC. ${ }^{22}$

\subsubsection{IRS}

IRS data are first processed through the SSC pipeline to remove instrumental artifacts, including dark current, droop signal, and flat-field structures. From these pipelined data, we proceed with the intermediate "droopres" data product. The SMART reduction package developed by the IRS Instrument Team at Cornell University (Higdon et al. 2004) is then used to extract the spectra. As a first step, we correct for the background emission and stray light by subtracting the images obtained from the two slit positions at which an object is observed for each module and order (automatically in standard starring mode). This results in a set of images containing a positive and negative spectrum in each observed order. Before extraction, all hot or dead pixels in each image are replaced.

For the spectral extraction, we use a straight-sided (boxcar) aperture limiting the extraction area around the positive source in the background-corrected images. Since all observations in the FEPS Legacy Program use high-accuracy peak-up, and the pointing of Spitzer is good to within 0"4 radius ( $1 \sigma)$, we fix the position of the aperture in each spectral order. The width of each aperture is chosen such that $99 \%$ of the source flux is within the aperture for all wavelengths in the order. A mean spectrum over all slit positions and cycles is computed for each individual order from the spectrum for each nod position and cycle. The orders are then combined. The quoted uncertainties are the $1 \sigma$ standard deviation of the distribution of data points used to calculate the mean spectrum over all cycles and nod positions, modified to include the errors of the photometric calibration. Finally, the orders are stitched together with unit weight by taking the mean flux at overlapping wavelength points.

\subsubsection{MIPS}

MIPS data were originally reduced using the Data Analysis Tool developed by the MIPS Instrument Team at the University of Arizona (Gordon et al. 2004), since the MIPS Instrument Team was responsible for the rapid development of the primary reduction algorithms. This package uses the raw data product available from the SSC data archive. Dark subtraction, scan mirror dependent flat field, electronic nonlinearity correction, droop subtraction, and cosmic-ray rejection are applied. For the final FEPS releases, the SSC pipeline products are used, since the combined efforts of the instrument team at the University of Arizona have been successfully implemented at the

\footnotetext{
${ }^{22}$ See http://ssc.spitzer.caltech.edu/irac/dh.
}

SSC, and the two reduction schemes have converged on a common, validated product.

Flux densities for each band are derived from aperture photometry. The position of the aperture was found by fitting a two-dimensional Gaussian to the core of the PSF when the object is detected. For nondetections, as is often the case for 70 and $160 \mu \mathrm{m}$, the aperture is centered on the object coordinates. Aperture correction factors are applied to match the "infinite aperture calibration" defined by the MIPS Instrument Team (see the MIPS Data Handbook ${ }^{23}$ ).

We use the standard deviation of the photometry from the stack of individual dither images $(24 \mu \mathrm{m})$, or the rms noise in the background propagated over the pixels in the object aperture as estimates of the random photometric uncertainty (70 and $160 \mu \mathrm{m})$. Total uncertainties are the RSS of the internal uncertainties and the published calibration uncertainties. As for IRAC, color corrections are not applied. However, we note that the MIPS team assumes a 10,000 K blackbody for its calibration (see footnote 23).

\subsubsection{Verification and Validation}

Quality control is applied to the Spitzer observing program as follows. All observations are verified, meaning checks on whether the correct source was observed, in the requested instrument mode, and following the prescribed AOR. The observations are further validated by considering photometric uncertainties derived from the observations, compared with theoretical uncertainties based on expected photon count rates and other known noise sources such as extragalactic confusion at 70 and $160 \mu \mathrm{m}$. Expected versus derived S/Ns are assessed for observations at wavelengths where the measured flux densities are consistent with being photospheric. Comparison with photospheric model expectations enables us to investigate systematic offsets in expected versus observed fluxes as a function of source color and brightness, although we are unable to unambiguously separate errors in the models from errors in the data. For wavelengths $\lesssim 24 \mu \mathrm{m}$, the exposure times were sufficient to measure the photospheric emission with expected $\mathrm{S} / \mathrm{N}>30$. We also verify that fluxes derived from different instruments over common wavelength ranges agree within the errors. Further details can be found in the Explanatory Supplements that accompany our data release through the SSC.

\subsection{Ancillary Data}

In addition to the Spitzer observations, we are engaged in a rich ancillary observing program that both complements and aids our interpretation of the Spitzer spectrophotometry. These data include ground-based $10 \mu \mathrm{m}$, submillimeter, and millimeter photometry; an echelle spectroscopic survey for photospheric characterization; and an AO imaging survey for companion detection and characterization.

\footnotetext{
${ }^{23}$ See http://ssc.spitzer.caltech.edu/mips/dh.
} 
1. Dust mass constraints.-We have engaged in a limited ground-based mid-infrared campaign on a few tens of FEPS targets. Imaging photometry at $10 \mu \mathrm{m}$ of selected members of the FEPS sample was obtained with the Long Wavelength Spectrometer (LWS) on the Keck I telescope, the SpectroCam-10 (SC-10) on the Hale $5 \mathrm{~m}$ telescope (Metchev et al. 2004), and also the Mid-Infrared Array Camera 3 (MIRAC3) on the Magellan I telescope (Mamajek et al. 2004). The observations were largely consistent with photospheric emission, with few exceptions where excess emission was detected, indicative of terrestrial zone dust. We also searched for dust located at larger radii and hence too cold to radiate strongly in the MIPS $160 \mu \mathrm{m}$ band. We obtained sub-mm (submillimeter) and/or mm continuum observations for approximately $\frac{1}{3}$ of our sample. Millimeter observations were obtained using the Owens Valley Radio Observatory (OVRO) millimeter-wave interferometer at $3.1 \mathrm{~mm}$ or the 37 element SIMBA bolometer camera on the $15 \mathrm{~m}$ Swedish-ESO Submillimetre Telescope (SEST) at $1.2 \mathrm{~mm}$, for a total of 89 stars. Sub-mm observations at $350 \mu \mathrm{m}$ were obtained for six stars using the SHARC bolometer camera on the $10.4 \mathrm{~m}$ telescope of the Caltech Submillimeter Observatory (CSO). These observations are discussed in detail by Carpenter et al. (2005). Thirteen FEPS sources were observed by Najita \& Williams (2005) at the James Clerk Maxwell Telescope (JCMT), using SCUBA. The sources were selected with an emphasis on those that are young (10 Myr to $300 \mathrm{Myr})$ and nearby $(<50 \mathrm{pc})$. Three sources were detected, including HD 107146 (Williams et al. 2004).

2. Gas mass constraints.-As a complement to our Spitzer $\mathrm{H}_{2}$ program, we are attempting $\mathrm{CO}$ rotational transition detection at millimeter wavelengths with OVRO, the Heinrich Hertz Submillimeter Telescope (HHSMT) facility of the Arizona Radio Observatory, and the JCMT, as well as fundamental vibrational emission at mid-infrared wavelengths $(4.5 \mu \mathrm{m})$ and the pure rotational transitions of $\mathrm{H}_{2}$ at $17 \mu \mathrm{m}$. A sample of $\sim 20$ sources was observed in the $\mathrm{CO}(2-1)$ line with the HHSMT, and upper limits were derived. These data are being combined with Spitzer observations, and results are reported in Pascucci et al. (2006). In addition, Najita \& Williams (2005) have searched for $\mathrm{CO}(3-2)$ emission from two of the sub-mm excess sources and placed limits on the gas mass in these systems. In the case of HD 107146, a conventional analysis suggests that the upper limit on the gas-to-dust ratio is much less than primordial.

3. Age diagnostics.-An important aspect of our program is determination of the tightest constraints possible on the ages of our sources (L. A. Hillenbrand et al. 2006, in preparation). To do so, we consider a number of diagnostics related to activity, which generally decreases with increasing stellar age, or elemental abundances, in particular Li I. We have assembled all X-ray information from the ROSAT archives. We have $R^{\prime} H K$ indices for over $\frac{3}{4}$ of our sample, from the literature or newly measured from our ancillary high-dispersion $(\mathcal{R} \approx 20,000$ from 3600 to $9500 \AA$ ) optical spectra. In addition to $\mathrm{Ca}$ II H and $\mathrm{K}$ core emission-line strengths, we are also measuring $\mathrm{H} \alpha$ emission/absorption equivalent widths, $\mathrm{Li}$ I equivalent widths, and rotational velocities, all of which change with stellar age. A full discussion of stellar age indicators and their likely uncertainties is beyond the scope of this paper. However, we find generally that when multiple age indicators are available for a given star, they agree with one another to better than 0.5 dex in log-age. This level of accuracy is adequate for investigating general trends in debris disk evolution. Finally, we can also derive effective temperatures, gravities, and metallicities for each star, through spectral synthesis modeling.

4. Stellar, substellar, and planetary mass companions.-In order to place our own solar system fully in context, we must consider the effects of stellar multiplicity at the same time we are considering dust disk evolution. Our Sun's planets exist at orbital radii ranging from 0.4 to $30 \mathrm{AU}$, with the Kuiper Belt extending out to at least $50 \mathrm{AU}$, and our Sun is not a member of a multiple star system. A significant fraction (30\%-80\%) of all Sun-like stars do appear to have been born in multiple star systems (binaries, triples, or quadruples; Mathieu et al. 2000) and, as shown for solar-type, solar-neighborhood multiples by Duquennoy \& Mayor (1991), the distribution of orbital periods is lognormal and peaked at 180 days or $30 \mathrm{AU}$, i.e., within our current solar system. Searches for companions (stellar, substellar, and planetary mass) to members of our Spitzer sample via both high-resolution imaging and spectroscopy are underway, in order to assess the role of multiplicity in disk evolution.

With the AO at the Palomar 200 inch $(5 \mathrm{~m})$ telescope, we have observed every northern star on our program with short $J H K_{s}$ exposures, designed to detect bright companions as close as $0{ }^{\prime \prime} 1$ (5 AU for sources at $50 \mathrm{pc}$ ). Such companions within the Spitzer beam ( $5^{\prime \prime}$ at $\left.24 \mu \mathrm{m}\right)$ are critical to account for when analyzing SEDs. For a selected subset of our stars, we are also performing deep $K_{s}$-band AO coronography designed to detect much fainter companions. Due to evolution of the massluminosity relationship and contrast limit, there is an intricate grid of trade-offs in the companion mass detection limit as a function of system age and orbital separation, with sensitivity to lower masses achievable at younger ages and larger separations. In the case of our target list, we are sensitive to masses as low as $3 M_{\text {Jup }}-10 M_{\text {Jup }}$ (for example, at separations of $2 "$ to $\Delta K=13 \mathrm{mag}$ at $\mathrm{S} / \mathrm{N}=5$ ). Follow-up proper motion, photometry, and spectroscopy are conducted with the Palomar 200 inch telescope and with Keck (e.g., Metchev \& Hillenbrand 2004, 2006).

High-dispersion spectroscopy is also being used to identify companions. Several double-line or single-line spectroscopic binaries have been found from our Palomar 60 inch $(1.52 \mathrm{~m})$ telescope echelle spectroscopy (White et al. 2006). In addition, at least $25 \%$ of our Spitzer target stars are located on various radial velocity planet search programs, a number of which are already known to have planetary companions or will be found with planetary companions over the next decade. Several FEPS 
targets are also being monitored photometrically in order to derive rotation periods from starspot activity on the stellar surface.

\section{SUPPORTING THEORETICAL FRAMEWORK}

Physical theories are needed in order to guide our observational program and to help interpret the results. The basic problem is to understand the formation and evolution of planetary systems based on observed SEDs and spectroscopic observations. In support of this work, we have undertaken a limited modeling effort aimed at constraining (1) basic disk properties, using a minimum of assumptions; (2) the amount of remnant gas in disks, based on IRS high-resolution spectra; and (3) the diversity of planetary architectures, based on estimates of geometric dust distributions derived from the SEDs.

\subsection{Toy Model for Solar System Evolution}

We start with a basic model of the evolution of our own solar system. As is well known, our planetary system contains two major debris belts: the Kuiper Belt and the asteroid belt, both of which are generating dust through mutual collisions of larger parent bodies. Figure 4 shows the dust mass and the observed SEDs predicted by two plausible models for the evolution of our solar system from $100 \mathrm{Myr}$ to $4.5 \mathrm{Gyr}$, as viewed from $30 \mathrm{pc}$. The models assume only a minimum mass solar nebula and planetesimal scattering and collision frequencies according to two simple analytic representations: one (Fig. 4, right) including the effects of dynamical instability postulated to have removed a large fraction of dust-producing parent bodies in the asteroid and Kuiper Belts in our solar system (Gomes et al. 2005; see also Strom et al. 2005), and one (Fig. 4, left) excluding those effects. In the former case, dust production diminishes linearly in time, as expected for a high-density planetestimal belt in collisional equilibrium, where dust is ultimately removed through radiation pressure blow-out of the smallest fragments (Dominik \& Decin 2003; Wyatt 2005) until the instability occurs at approximately 500-600 Myr. In the latter case, the belt grinds itself down to the low-density limit where dust removal is dominated by Poynting-Robertson (P-R) drag and the dust mass observed decays at $t^{-2}$. In the absence of this instability, our hypothetical solar system is detectable by Spitzer with IRAC (zodiacal dust disk) and MIPS (Kuiper disk) from $30 \mathrm{pc}$ at age $100 \mathrm{Myr}$, while only the Kuiper Belt dust would be found at an age of 4.5 Gyr. In this way, we can attempt to address whether or not our solar system is common or rare compared to typical stars in the disk of the Milky Way. Details concerning this model can be found in Meyer et al. (2006).

One deficiency in this model is neglecting the drag on orbiting grains due to stellar winds. Azimuthal wind drag could dominate radiation P-R drag for the high mass-loss rates expected from young solar-type stars (Jura 2004). This would decrease grain lifetimes in systems that are P-R-drag dominated and diminish the number of systems thought to be collisionally dominated. However, recent work (Wood et al. 2005) suggests that the large effects suggested in earlier work (Wood et al. 2002) may have been overestimated. While this effect can be important, its magnitude is still uncertain.

\subsection{Constraints on Dust Properties}

The observed SEDs from dust disks are determined in part by the optical properties of the dust contained therein. Both particle size and composition are important in determining the absorption and emission properties of the dust, thus determining its temperature for a given distance from the central star. In the absence of constraints on dust properties from spectral features, resolved images of disks in thermal emission or scattered light, or far-IR/sub-mm spectral slopes, models to explain the observed SEDs of debris disks are necessarily degenerate (see, e.g., Hines et al. 2006). Spectroscopic observations of solid state features can provide important constraints on physical models for the dust. For example, large particles $\left(a_{\text {dust }}>\lambda_{\mathrm{rf}} /\right.$ $\pi)$ are not efficient radiators at their natural resonance frequencies. Thus, the absence of expected solid state features from abundant species can indicate a minimum grain size. Similarly, specific chemical compositions of dust can change the shape and central wavelength of resonance features or indicate significant structural differences in the dust (e.g., amorphous vs. crystalline silicates). Discerning the difference between Mgand Fe-rich end-member silicates and fractions of amorphous to crystalline silicates provides crucial information concerning the chemical properties of the nebula in which parent body planetesimals formed, as well as the processing history of the dust. Detailed models exploring these effects are described in Wolf \& Hillenbrand (2003) and J. Bouwman et al. (2006, in preparation).

\subsection{Dynamics of Disk-Planet Interactions}

As part of our theory effort, we have developed numerical tools to model debris disks originating from an outer belt of planetesimals and evolving under the effect of gravitational perturbation from giant planets in various planetary configurations and for different dust particle sizes and compositions (Moro-Martín \& Malhotra 2002, 2003, 2005; Moro-Martín et al. 2005). Although the particle dynamics is chaotic, our method can robustly estimate the equilibrium radial density distribution of dust. The dust density structure carved by giant planets affects the shape of the disk SED, in a manner that depends on the mass and location of the perturbing planet as well as the grain properties (chemical composition, density, and size distribution). We found that the SED of a debris disk with embedded giant planets is fundamentally different from that of a disk without planets, the former showing a significant decrease of the near-/mid-IR flux due to the clearing of dust inside the planet's orbit. The SED is particularly sensitive to the location of the planet, i.e., to the area inside the planet's 

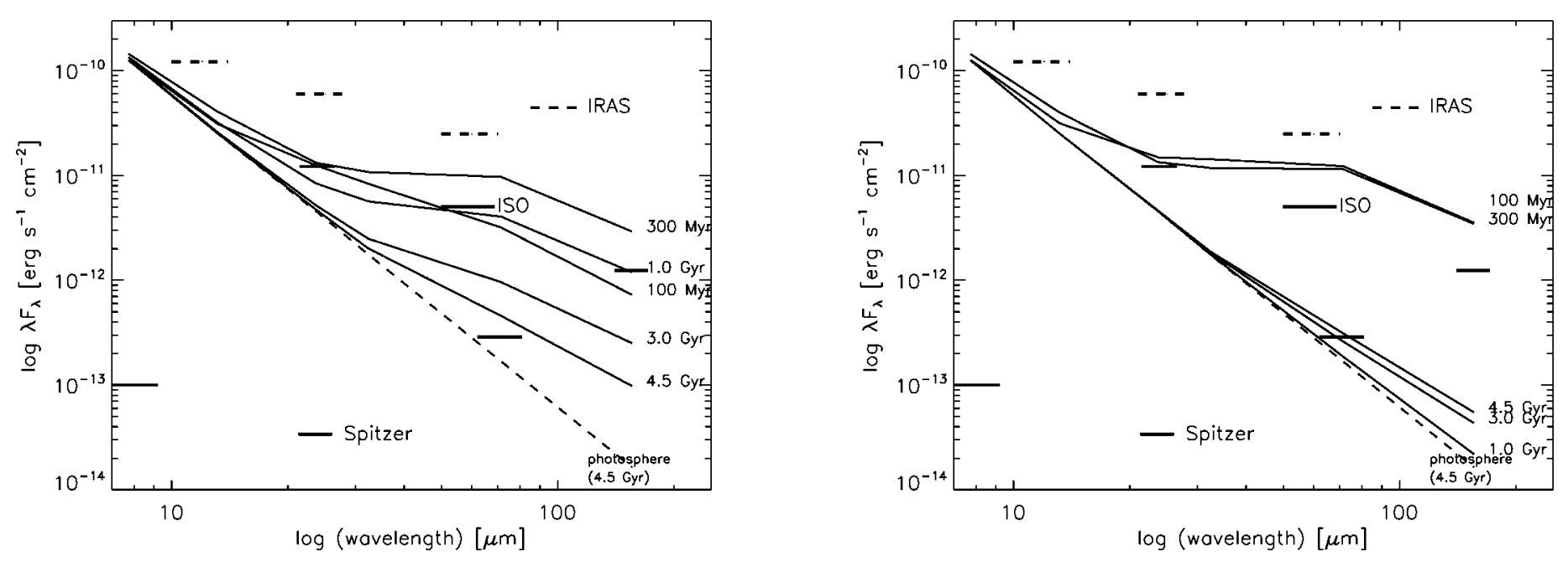

FIG. 4.- Toy model for the evolution of our solar system debris disk surrounding the Sun, as observed from a distance of $30 \mathrm{pc}$. The model shown in the left panel includes continuous removal of planetesimals starting from the minimum mass solar nebula in solids and evolving toward the present day, without any dramatic clearing event, such as the late heavy bombardment. The model shown in the right panel begins with the minimum mass solar nebula in solids, as in the left panel, but includes a dramatic clearing event, such as the late heavy bombardment, between 300 Myr and 1 Gyr, in accordance with recent models of Gomes et al. (2005; see also Strom et al. 2005).

orbit that is depleted in dust due to gravitational scattering by the planet. Our dynamical models show that for a planet in a circular orbit with semimajor $a_{\mathrm{p}}$, the radius of the depleted inner zone is in the range of $(0.8-1.2) a_{\mathrm{pl}}$, depending on the planet mass. Our models also show that the dust depletion factor (i.e., the ratio between the dust density inside and outside the depleted region) depends significantly on the planet mass when the mass is in the range $1 M_{\mathrm{Nep}}<M_{\mathrm{pl}}<3 M_{\mathrm{Jup}}$. However, there are two issues that complicate the interpretation of the SED in terms of planet location:

1. The SEDs are degenerate. In particular, there is a degeneracy between the dust grain chemical composition and the semimajor axis of the planet responsible for the inner gap. For example, the SED of a dust disk dominated by weakly absorbing grains (Fe-poor silicate) has its minimum at wavelengths longer than those of a disk dominated by strongly absorbing grains (e.g., carbonaceous and Fe-rich silicate), which may be mistaken by the presence of a larger inner gap. This degeneracy can be resolved with either high-resolution spectroscopy (which would constrain the grain chemical composition as discussed above) or high spatial resolution images (which would spatially resolve the inner edge of the dust disk).

2. Because of the Spitzer sensitivity limit, the debris disks observed by FEPS may be in the collision-dominated regime, where the dynamics of the dust particles is dominated by collisions rather than P-R drag (Dominik \& Decin 2003; Wyatt 2005). This may result in the dust particles suffering multiple collisions, which could break them down into smaller and smaller grains until they are blown out from the system by radiation pressure, before they have time to migrate from the dust-producing planetesimals to a planet-crossing orbit.

\subsection{Models of Gas in Disks}

In order to interpret our high-resolution spectroscopic observations of gas in disks from ages of 3 to $100 \mathrm{Myr}$, we have developed detailed thermochemical models of gas and dust in optically thin disks (Gorti \& Hollenbach 2004). Our models calculate the gas spectral line emission and dust continuum emission for comparison with observed data and thereby infer disk properties. The models calculate the gas and dust temperatures separately, assuming a balance between the heating and cooling processes. We include various heating sources for the gas, such as collisions with warm dust heated by stellar radiation, X-rays, exothermic chemical and photo reactions, cosmic rays, and grain photoelectric heating. The gas cools by ionic, atomic, and molecular line emission. The disk temperature structure, vertical density structure, and chemistry are self-consistently calculated in our disk models by requiring thermal balance and vertical pressure equilibrium, and by assuming steady state chemistry. Our chemical network consists of 73 ionic, atomic, and molecular species involving $\mathrm{H}, \mathrm{He}$, $\mathrm{C}, \mathrm{O}, \mathrm{Si}, \mathrm{Mg}, \mathrm{Fe}$, and $\mathrm{S}$, and 537 reactions. Inputs to our models are stellar parameters such as the radiation field at X-ray, UV, and visible wavelengths; the disk surface density distribution in gas and dust; and dust properties (chemical composition and size distribution). Most of the stellar and dust parameters are determined through ancillary observations and by modeling of the dust continuum observed through the FEPS program. For a given dust and gas surface density distribution, our theoretical models can predict the spectral line emission from the gas and the dust continuum emission for comparison to observations.

We are in the process of developing similar gas disk models 
for younger, optically thick dust disks. We use a two-layer model for the dust-temperature calculation (Chiang \& Goldreich 1997) and adopt a procedure similar to that for optically thin disks for the gas temperature. The gas and dust temperature are calculated separately, and the emergent line plus continuum spectrum is computed.

Spitzer IRS in the high-resolution mode is capable of detecting $(3 \sigma)$ warm gas $\left(T_{\text {gas }} \sim 100 \mathrm{~K}\right)$ masses of $\lesssim 0.2 M_{\text {Jup }}$ in disks at distances of 160 pc or less (Gorti \& Hollenbach 2004). The FEPS $\mathrm{H}_{2}$ program will measure spectral line fluxes (or upper limits to line fluxes), and these will be compared with the gas models to infer the gas masses in disks and the spatial distribution of gas (e.g., Hollenbach et al. 2005; Pascucci et al. 2006).

\section{ANALYSIS PLAN}

Our approach to analyzing the data collected as part of FEPS starts with as few assumptions concerning the physical nature of the observed system as possible, and proceeds to more elaborate models concerning the excess emission. At each stage, additional assumptions are made, which enable a richer interpretation of the data. However, the certainty of our conclusions diminishes as we proceed. In any event, we attempt to clearly state our assumptions as we go, and we try to be careful not to proceed on the basis of assumptions that are demonstrably false. In following this analysis procedure, it is important to always keep in mind how the results depend on the input assumptions, as well as explore the full range of model parameters allowed by the data, including the uncertainties.

Emission in excess of that expected from the stellar photospheres is found by subtracting model photospheric flux estimates from the observed fluxes across the wavelength range accessible to Spitzer. We execute the following generic analysis for all sources with excess emission of $5 \sigma$ or greater at one or more wavelengths, or equivalent detections of lower $\mathrm{S} / \mathrm{N}$ but at two or more wavelengths. We begin by calculating an approximate dust temperature if the excess is detected at two or more wavelengths. If the excess is detected at only one wavelength, we derive a temperature limit using the excess and the bluest broadband point without excess. With this temperature fit to the data, we then estimate the ratio of excess luminosity in the infrared to the total stellar luminosity $f \sim f_{\text {IRX }} / f_{*}$.

If the source presents an excess over a broad range of wavelength, we explore whether a range of dust temperatures would be a more appropriate model. Emission that appears to be optically thick in the direction perpendicular to the disk from the point of view of the observer over a range of wavelength is initially assumed to be a primordial gas-rich disk left over from the formation of the star. If high-resolution IRS data are available for the source, we can assess whether these observations constrain the amount of molecular gas remnant in the disk. Other observations, such as mm-wave $\mathrm{CO}$ data or evidence for active accretion onto the star, could also suggest a primordial disk. Evidence for a flared disk geometry from the SED could also provide evidence for a gas-rich primordial disk.

If there is no evidence for remnant gas in the disk, we proceed under the premise that the disk might be a debris disk, where the dust we see is generated through collisions of larger parent bodies in a planetesimal belt. Assuming that the grains are large, efficient absorbers and emitters of light, we can calculate the required dust cross-sectional area for the emitting grains and determine a plausible radius in the disk for the planetesimal belt. For an excess observed at a wavelength $\lambda$, we assume that grains larger than $\lambda / \pi$ can be treated approximately as perfect blackbodies. Given the luminosity of the star, we can also calculate the radiation pressure blow-out size, providing a bound on the smallest particles that could be responsible for the radiation. To further constrain the grain size distribution and composition, we also search for evidence of solid state emission features in the IRS low-resolution spectra obtained for each source. If grains exist in the circumstellar disk at temperatures corresponding to emission at the appropriate wavelength (e.g., $300 \mathrm{~K}$ for the $10 \mu \mathrm{m}$ silicate feature), the lack of expected spectral features attributed to specific grain compositions can place constraints on their abundance (e.g., the upper limit to the fraction of crystalline silicates in the debris dust) or particle size (for the solid state, grains larger than $\lambda_{\mathrm{rf}} / \pi$ will not be efficient radiators in the resonance feature) or both. These observations, as well as the observed spectral slope in the far-infrared/sub-mm, constrain the grainsize distribution and thus the range of plausible models for the radii in the disk responsible for the emission at a given temperature. For example, very small grains $(<0.1 \mu \mathrm{m})$ can reach the same temperature at $>30 \mathrm{AU}$ as very large grains $(>10 \mu \mathrm{m})$ at $<3$ AU (Backman \& Paresce 1993).

Armed with this information, we can calculate basic quantities such as the mass surface density of emitting grains for a given particle size/radius in the disk. This enables us to compare the lifetime of grains of various size under the assumption that they are subject to both mutual collisions in the disk and the effects of P-R drag (e.g., Burns et al. 1979). In most cases, observed debris disks have surface densities so high that many collisions will occur between dust grains before they have time to evolve in a radius significantly under P-R drag (Dominik \& Decin 2003; Wyatt 2005). In this case, the grains are eroded down to the blow-out size and removed from the system through radiation pressure. If the IR excess is confined to cooler temperatures (and therefore larger radii), we can also place limits on the mass surface density inside of $R_{\text {inner }}$ and characterize the presence of an inner hole in the dust distribution. In principle, limits on the mass surface density outside of the observed disk radius and $R_{\text {outer }}$ could be constrained by FIR and sub-mm observations as well.

In the case where the IR excess is detected with $\mathrm{S} / \mathrm{N}>5$ at several wavelengths, we explore disk model parameters in a robust way by modeling the excess emission and calculating the best fit by computing the reduced $\chi^{2}$ metric. Given the 
number of degrees of freedom in the model, the number of observations, and robust uncertainties in the observations, the reduced $\chi^{2}$ can provide an estimate of the probability that any parameter lies within a range of values (confidence interval) if the data were drawn from a particular model. As a result, we can define contours of allowed (correlated) parameters in a multidimensional space defined by the model parameters. This requires clearly defined model parameters that can be varied over a range to produce acceptable fits to the data, as well as clearly defined uncertainties in our data that can be interpreted as errors in a Gaussian probabilistic sense.

Even in the absence of robust parameter estimation as described above, we can often constrain the family of permitted models or offer a preferred model on the basis of likely physical scenarios and Occam's razor. For example, in the case where two models match well the observed excess (small grains at large radii vs. large grains at smaller radii), one can argue that the large grain model might be preferred if the surface density of that model were so large as to enable the disk to maintain an inner hole on the basis of mutual collisions down to the blow-out size. The small grain at large radii model might have a surface density so low that an interior planet might be required in order to avoid grains filling in the hole under the action of P-R drag. Thus, the large grain model at small radii requires fewer assumptions and might be preferred. Furthermore, we know that $\mathrm{T}$ Tauri stars in their youth have optically thick circumstellar disks ranging from less than $0.1 \mathrm{AU}$ to greater than $30 \mathrm{AU}$. It might seem implausible to require a massive circumstellar disk composed entirely of small grains less than $1 \mu \mathrm{m}$ at $R>100 \mathrm{AU}$ with no evidence for dust or planetesimals inside of $30 \mathrm{AU}$, rather than a more modest remnant disk composed of larger grains at radii where we know giant planets form and disks exist around Sun-like stars (see Kim et al. 2005; Hines et al. 2006).

Finally, all of the models considered should make specific predictions that can be tested with follow-up observations. For example, scattered-light imaging with AO on large groundbased telescopes, or using coronagraphy on the Hubble Space Telescope, could provide constraints on the radial extent of small grains in the debris disk systems. More sensitive Spitzer observations at mid- and far-IR wavelengths could improve on low-S/N initial detections. Sub-mm observations can constrain disk models - in particular, the use of interferometers to resolve the FIR/sub-mm emission initially detected by Spitzer. In general, having resolved images of disks, at one or more wavelengths in thermal emission or in scattered light, breaks many of the degeneracies associated with SED modeling. For the nearest, youngest systems in which a hole in the dust distribution is inferred from SED modeling, we can test those predictions using highcontrast imaging techniques to search for warm massive planets in the circumstellar environment $R>5 \mathrm{AU}$. The connection of dust disk emission with the presence/absence of giant planets is still poorly understood (Moro-Martín et al. 2006; Beichman et al. 2005) and is one of the key goals of the FEPS Legacy Science Program.

\section{FEPS DATA PRODUCTS}

As part of our commitment to the Spitzer Legacy Science Program, we plan to deliver high-quality enhanced data products for the benefit of the community, as well as documentation that will enable archival researchers to utilize these data in the most efficient way possible. In addition to ground-based ancillary data, and the Spitzer database itself, we also provide resources that enable careful scrutiny of the Spitzer calibration, as well as tools for the research community interested in interpreting observations of debris disk systems.

\subsection{Ancillary Data Products}

Ancillary data collected in support of FEPS are provided via the published literature and include all data discussed above:

1. $10 \mu \mathrm{m}$ photometry (e.g., Metchev et al. 2004; Mamajek et al. 2004).

2. Sub-mm and mm continuum photometry (e.g., Williams et al. 2004; Carpenter et al. 2005; Najita \& Williams 2005).

3. CO gas line measurements or upper limits (e.g., Najita \& Williams 2005; Pascucci et al. 2006).

4. Stellar age indices (e.g., L. A. Hillenbrand et al. 2006, in preparation).

5. Stellar photospheric properties (provided through Legacy deliveries to the SSC; see $\S 6.3$ ).

6. Detected companion properties and limits on undetected companions (e.g., Metchev \& Hillenbrand 2004, 2006).

Selected data tables from these sources are also included in the Spitzer Legacy Science archive (in particular, the midinfrared and sub-mm survey data and those data used in fitting photospheric parameters).

\subsection{Spitzer Data Products}

Spitzer data are provided to the SSC for all 328 stars in the FEPS sample as raw and SSC pipelined products (accessed through Leopard) and as "enhanced data products" (see footnote 21). A pointed observations photometric catalog (POPC) is available, consisting of flux densities for $\operatorname{IRAC}(3.6,4.5$, and $8.0 \mu \mathrm{m}$ ) and MIPS (24 and $70 \mu \mathrm{m}$ ) observations for all sources. IRAC $5.8 \mu \mathrm{m}$ flux densities are available for five stars that were part of the early validation portion of the program. Due to time constraints imposed by slight modifications in the expected $\mathrm{S} / \mathrm{N}$, based on the updated on-orbit performance of the instruments, we chose to drop the $5.8 \mu \mathrm{m}$ band from our general survey rather than decrease the number of targets. ${ }^{24}$ Similarly, $160 \mu \mathrm{m}$ observations are available for approximately $10 \%$

\footnotetext{
${ }^{24}$ Because FEPS used the subarray mode of IRAC observations reserved for bright stars, we do not obtain $5.8 \mu \mathrm{m}$ observations simultaneously with the $3.6 \mu \mathrm{m}$ observations, as is the usual case.
} 
of the FEPS sample. The confusion limit at $160 \mu \mathrm{m}$ for most of our targets was well above the sensitivity level needed to test our toy model for the evolution of our solar system through observation of Sun-like stars from 3 Myr to 3 Gyr. Therefore, we chose a limited campaign of $160 \mu \mathrm{m}$ observations in order to preserve the "discovery space" (more than 30 times, compared to the ISO at these wavelengths) enabled by MIPS $160 \mu \mathrm{m}$ observations for a random subset of our sample. We also include an image atlas, based on mosaicked IRAC and MIPS images. In the case of the MIPS 70 and $160 \mu \mathrm{m}$ observations, these images represent the data from which the photometry in the POPC is derived. In the case of the IRAC and MIPS $24 \mu \mathrm{m}$ data, photometry is derived from individual frames, and the results in the POPC are the median values with associated errors, as described above.

Low-resolution ( $\mathcal{R} \sim 64-128)$ spectra obtained with the IRS are presented in the spectral atlas comprised of extracted spectra from 7.4 to $33 \mu \mathrm{m}$ for all stars, and spectra from 5.2 to $33 \mu \mathrm{m}$ for 3-30 Myr stars. Again, because of on-orbit sensitivities, we chose to drop the second order of the short-low module (providing spectra from 5.2 to $7.4 \mu \mathrm{m}$ ) for the older sources in the sample, rather than decrease the number of stars in our program. We also plan to deliver an atlas of high-resolution $(\mathcal{R} \sim 600)$ IRS spectra, comprised of data from 9.9 to $37.2 \mu \mathrm{m}$ for 35 stars chosen from among our full sample. Tables of emission-line fluxes (or upper limits) are provided for six features selected as most sensitive to remnant gas, based on the models of Gorti \& Hollenbach (2004).

\subsection{Calibration Products}

A primary product delivered for all sources in the survey is models of the stellar photosphere fit to short-wavelength photometry and extended through the Spitzer wavelengths. The photospheric emission component is modeled by fitting Kurucz atmospheres, including convective overshoot, to available $B V$ Johnson, vby Strömgren, $B_{T} V_{T}$ Tycho, $H_{p}$ Hipparcos, $R I$ Cousins, and $J H K_{s}$ 2MASS photometry. Predicted magnitudes were computed as described in Cohen et al. (2003 and references therein), using the combined system response of filter, atmosphere (for ground-based observations), and detector. The bestfit Kurucz model was computed in a least-squares sense with the effective temperature and normalization constant (i.e., solid angle of the source physically set by the distance and radius) as free parameters, $[\mathrm{Fe} / \mathrm{H}]$ fixed to solar metallicity, and surface gravity fixed to the value appropriate for the adopted stellar age and mass. Visual extinction is fixed to $A_{V}=0^{m}$ for stars with distances less than $40 \mathrm{pc}$, assumed to be within the dustfree Local Bubble, but a free parameter for stars at larger distances. A file containing the best-fit stellar SED is provided along with associated uncertainties in the fitted parameters. These fits are available from the Spitzer Legacy Science Archive (see footnote 21).

Our secondary products include reported information con- cerning instrument calibration based on observations of FEPS targets that are consistent with the expected level of stellar photospheric emission. We also investigate the consistency between different instruments, such as (1) IRAC photometry at 5.4 and $8.0 \mu \mathrm{m}$ and the IRS spectra from 5.2 to $10 \mu \mathrm{m},(2)$ MIPS $24 \mu \mathrm{m}$ photometry and IRS spectra from 20 to $26 \mu \mathrm{m}$, and (3) low- and high-resolution spectra from 9.9 to $33 \mu \mathrm{m}$. Details concerning these diagnostic comparisons can be found in the FEPS Explanatory Supplements that accompany our data releases to the SSC.

\section{SUMMARY OF RESULTS TO DATE}

We briefly summarize the results from the FEPS program to date. Silverstone et al. (2006) report a search for warm dust excesses surrounding 74 Sun-like stars with ages 3-30 Myr. Only five objects show evidence for excess emission between 3.6 and $8 \mu \mathrm{m}$. All appear to be optically thick disks, and four show signs of active accretion from the disk onto the star. This result suggests that the transition time between optically thick to optically thin inside of $1 \mathrm{AU}$ is very short $(<1 \mathrm{Myr})$. J. Bouwman et al. (2006, in preparation) analyze the dust size and composition in the optically thick accretion disks in the FEPS sample from IRS high-resolution observations. They report a correlation between the inferred grain size and slope of the SED tracing disk structure. They also analyze the contribution of crystalline silicate emission to the observed spectra, comparing the results to models for the production of crystalline grains in the disk. Hollenbach et al. (2005) report analysis of the IRS high-resolution data for HD 105 (30 Myr old), indicating that less than $0.1 M_{\text {Jup }}$ of gas persists between 1 and 40 AU. Extending this work, Pascucci et al. (2006) report similar results for a sample of 15 stars spanning a range of age from 3 to 100 Myr. It appears that gas-rich disks capable of forming Jupiter-mass planets dissipate in less than $10 \mathrm{Myr}$. Additional planned observations will address whether gas-rich disks persist beyond $3 \mathrm{Myr}$.

Hines et al. (2006) report the discovery of an unusual warm debris disk around the 30 Myr old star HD 12039. Assuming the excess is produced from dust dominated by large blackbody grains, the emitting area is estimated to be between 4 and 6 AU from the star, comparable to the location of our own asteroidal debris belt. Stauffer et al. (2005) analyze the frequency of $24 \mu \mathrm{m}$ excess emission among Sun-like stars in the 100 Myr old Pleiades open cluster. They find that a small fraction of stars exhibit excess emission attributable to warm dust in the terrestrial planet zone. Future work will assess the fraction of warm dust excess as a function of age throughout the FEPS sample.

Initial discoveries of cool dust debris (Meyer et al. 2004; Kim et al. 2005) around FEPS targets suggest that (1) there is a large dispersion of inferred dust masses in outer debris belts at any given time, (2) there is a general trend of less dust at later times, and (3) most of these systems have large inner holes in their radial dust distributions. Inner holes of order 
$30 \mathrm{AU}$ in these disks with large dust mass surface density are probably maintained by mutual collisions of grains whose sizes are diminished down to the blow-out size, at which time they are removed from the system due to radiation pressure (Dominik \& Decin 2003; Wyatt 2005), although we cannot rule out the presence of gas giant planets in most systems. In an analysis of a possible correlation between the presence of debris and radial velocity planets, Moro-Martin et al. (2006) report no correlation in the FEPS database or in the published surveys of Bryden et al. (2006), as well as the detection of a debris disk surrounding planet host star HD 38529. Future work will focus on the fraction of objects with excess emission, the evolution in the mean dust mass as a function of age, and the presence of extended debris disks around some stars (L. A. Hillenbrand et al. 2006, in preparation), as well as connections between the presence of debris and metallicity of the central star.
We would like to thank our colleagues at mission control at the Jet Propulsion Laboratory and at the Spitzer Science Center, as well as members of the IRAC, IRS, and MIPS instrument teams for their contributions to this work. This work is based in part on observations made with the Spitzer Space Telescope, which is operated by the Jet Propulsion Laboratory, California Institute of Technology, under NASA contract 1407. FEPS is pleased to acknowledge support through NASA contracts 1224768,1224634 , and 1224566 administered through the JPL. M. R. M. is also supported through membership in NASA's Astrobiology Institute. S. W. was supported by the German Research Foundation (DFG) through Emmy Noether grant WO $857 / 2-1$. E. E. M. is supported by a Clay Fellowship from the Smithsonian Astrophysical Observatory. The MPIA team is supported through the European Planet Formation Network, funded by the European Union.

\section{REFERENCES}

Backman, D. E., \& Paresce, F. 1993, in Protostars and Planets III, ed. E. H. Levy \& J. I. Lunine (Tucson: Univ. Arizona Press), 1253 Beckwith, S. V. W., \& Sargent, A. I. 1996, Nature, 383, 139

Beichman, C. A., et al. 2005, ApJ, 622, 1160

Benjamin, R. A., et al. 2003, PASP, 115, 953

Bryden, G., et al. 2006, ApJ, 636, 1098

Burns, J. A., Lamy, P. L., \& Soter, S. 1979, Icarus, 40, 1

Carpenter, J. M., Wolf, S., Schreyer, K., Launhardt, R., \& Henning, T. 2005, AJ, 129, 1049

Chiang, E. I., \& Goldreich, P. 1997, ApJ, 490, 368

Cohen, M., Megeath, S. T., Hammersley, P. L., Martín-Luis, F., \& Stauffer, J. 2003, AJ, 125, 2645

de Muizon, M. J. 2005, Space Sci. Rev., 119, 201

Dickinson, M., Giavalisco, M., \& The GOODS Team. 2003, in The Mass of Galaxies at Low and High Redshift, ed. R. Bender \& A. Renzini (Garching: Springer), 324

Dominik, C., \& Decin, G. 2003, ApJ, 598, 626

Duquennoy, A., \& Mayor, M. 1991, A\&A, 248, 485

Evans, N. J., et al. 2003, PASP, 115, 965

Fazio, G. G., et al. 2004, ApJS, 154, 10

Gomes, R., Levison, H. F., Tsiganis, K., \& Morbidelli, A. 2005, Nature, 435, 466

Gordon, K. D., et al. 2004, Proc. SPIE, 5487, 177

Gorti, U., \& Hollenbach, D. 2004, ApJ, 613, 424

Greaves, J. S., et al. 1998, ApJ, 506, L133

Guillout, P., Sterzik, M. F., Schmitt, J. H. M. M., Motch, C., \& Neuhaeuser, R. 1998, A\&A, 337, 113

Henry, T. J., Soderblom, D. R., Donahue, R. A., \& Baliunas, S. L. 1996, AJ, 111, 439

Higdon, S. J. U., et al. 2004, PASP, 116, 975

Hines, D. C., et al. 2006, ApJ, 638, 1070

Hollenbach, D., et al. 2005, ApJ, 631, 1180

Houck, J. R., et al. 2004, ApJS, 154, 18

Jura, M. 2004, ApJ, 603, 729

Kalas, P., Liu, M. C., \& Matthews, B. C. 2004, Science, 303, 1990

Kennicutt, R. C., et al. 2003, PASP, 115, 928

Kim, J. S., et al. 2005, ApJ, 632, 659

Lagrange, A.-M., Backman, D. E., \& Artymowicz, P. 2000, in Protostars and Planets IV, ed. V. Mannings, A. P. Boss, \& S. S. Russell (Tucson: Univ. Arizona Press), 639
Lonsdale, C. J., et al. 2003, PASP, 115, 897

Lorenzetti, D. 2005, Space Sci. Rev., 119, 181

Mamajek, E. E., Meyer, M. R., Hinz, P. M., Hoffmann, W. F., Cohen, M., \& Hora, J. L. 2004, ApJ, 612, 496

Mamajek, E. E., Meyer, M. R., \& Liebert, J. 2002, AJ, 124, 1670

Marcy, G., Butler, R. P., Fischer, D., Vogt, S., Wright, J. T., Tinney, C. G., \& Jones, H. R. A. 2005, Prog. Theor. Phys. Suppl., 158, 24

Mathieu, R. D., Ghez, A. M., Jensen, E. L. N., \& Simon, M. 2000, in Protostars and Planets IV, ed. V. Mannings, A. P. Boss, \& S. S. Russell (Tucson: Univ. Arizona Press), 703

Metchev, S. A., \& Hillenbrand, L. A. 2004, ApJ, 617, 1330

- 2006, ApJ, 651, 1166

Metchev, S. A., Hillenbrand, L. A., \& Meyer, M. R. 2004, ApJ, 600, 435

Meyer, M. R., Backman, D. E., Weinberger, A., \& Wyatt, M. 2006, in Protostars and Planets V, ed. B. Reipurth, D. Jewitt, \& K. Keil (Tucson: Univ. Arizona Press), in press

Meyer, M. R., et al. 2004, ApJS, 154, 422

Miyake, K., \& Nakagawa, Y. 1993, Icarus, 106, 20

Molster, F., \& Kemper, C. 2005, Space Sci. Rev., 119, 3

Moro-Martín, A., \& Malhotra, R. 2002, AJ, 124, 2305 . 2003, AJ, 125, 2255 2005, ApJ, 633, 1150

Moro-Martín, A., Wolf, S., \& Malhotra, R. 2005, ApJ, 621, 1079

Moro-Martín, A., et al. 2006, ApJ, in press

Najita, J., \& Williams, J. P. 2005, ApJ, 635, 625

Pascucci, I., et al. 2006, ApJ, 651, 1177

Rieke, G. H., et al. 2004, ApJS, 154, 25

Silverstone, M. D., et al. 2006, ApJ, 639, 1138

Soderblom, D. R., \& King, J. R. 1999, in ASP Conf. Ser. 185, Precise Stellar Radial Velocities, ed. J. B. Hearnshaw \& C. D. Scarfe (San Francisco: ASP), 102

Stauffer, J. R., et al. 2005, AJ, 130, 1834

Strom, R. G., Malhotra, R., Ito, T., Yoshida, F., \& Kring, D. A. 2005, Science, 309, 1847

Torra, J., Fernández, D., Figueras, F., \& Comerón, F. 2000, Ap\&SS, 272, 109

Weinberger, A. J., Becklin, E. E., Schneider, G., Smith, B. A., Lowrance, P. J., Silverstone, M. D., Zuckerman, B., \& Terrile, R. J. 1999, ApJ, 525, L53 
Werner, M. W., et al. 2004, ApJS, 154, 1

White, R., Gabor, J., \& Hillenbrand, L. 2006, AJ, in press

Williams, J. P., Najita, J., Liu, M. C., Bottinelli, S., Carpenter, J. M., Hillenbrand, L. A., Meyer, M. R., \& Soderblom, D. R. 2004, ApJ, 604,414

Wolf, S., \& Hillenbrand, L. A. 2003, ApJ, 596, 603
Wood, B. E., Müller, H.-R., Zank, G. P., \& Linsky, J. L. 2002, ApJ, 574,412

Wood, B. E., Müller, H.-R., Zank, G. P., Linsky, J. L., \& Redfield, S. 2005, ApJ, 628, L143

Wyatt, M. C. 2005, A\&A, 433, 1007 